\title{
Decomposition of the Electronic Energy in Terms of Density, Density Coherence, and the Connected Part of the Two-Body Reduced Density Matrix
}

\author{
Dayou Zhang and Donald G. Truhlar* \\ Department of Chemistry, Chemical Theory Center, and Minnesota Supercomputing Institute, \\ University of Minnesota, Minneapolis, Minnesota 55455-0431, United States
}

TABLE OF CONTENTS

Figure $\mathrm{S} 1 \quad$ Dissociation curves of $\mathrm{N}_{2}$ using the DCC decomposition for RHF and MRCI

Figure S2 Dissociation curves of $\mathrm{N}_{2}$ using the DCC decomposition for RHF and CASSCF

Figure S3 Dissociation curves of $\mathrm{N}_{2}$ using the DCC decomposition for CASSCF and MRCI

Table S1 Absolute energies in hartrees of the DCC decomposition for $\mathrm{H}_{2}$

S-4

Table S2 Absolute energies in hartrees of the DCC decomposition for $\mathrm{F}_{2}$

Table S3 Absolute energies in hartrees of the DCC decomposition for $\mathrm{N}_{2}$

S-10

Table $44 \quad$ Absolute energies in hartrees of the $\mathrm{CN}$ decomposition for $\mathrm{H}_{2}$

S-13

Table S

Absolute energies in hartrees of the $\mathrm{CN}$ decomposition for $\mathrm{F}_{2}$

S-16

Table S6

Absolute energies in hartrees of the $\mathrm{CN}$ decomposition for $\mathrm{N}_{2}$

S-19

Sample input file

$\mathrm{S}-22$

Key modifications to the OpenMolcas source code and the Python script 

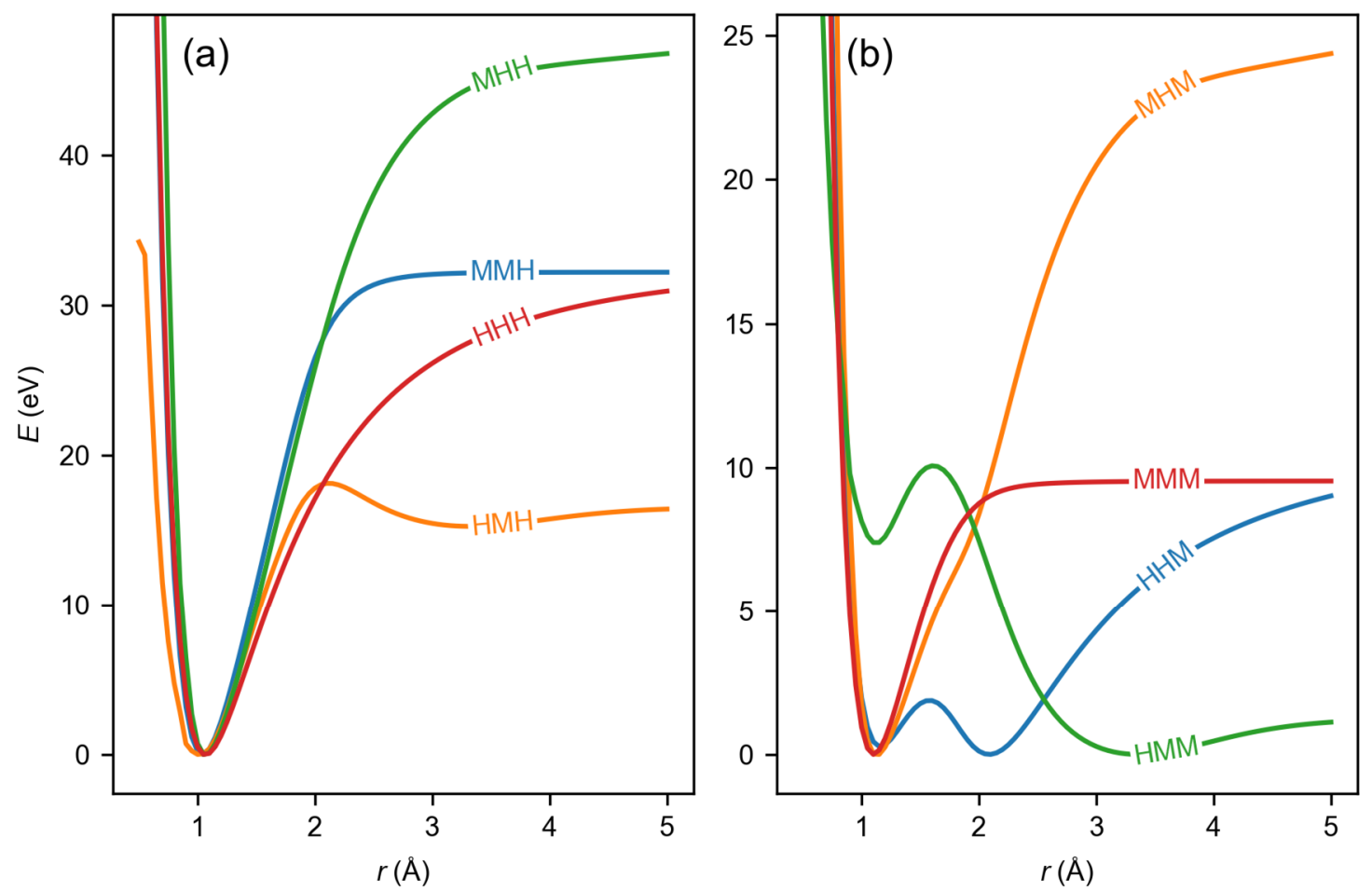

Figure S1. Dissociation curves of $\mathrm{N}_{2}$ using the DCC decomposition for RHF and MRCI. (a) combinations with $E^{\text {con }}$ from RHF. (b) combinations with $E^{\text {con }}$ from MRCI.
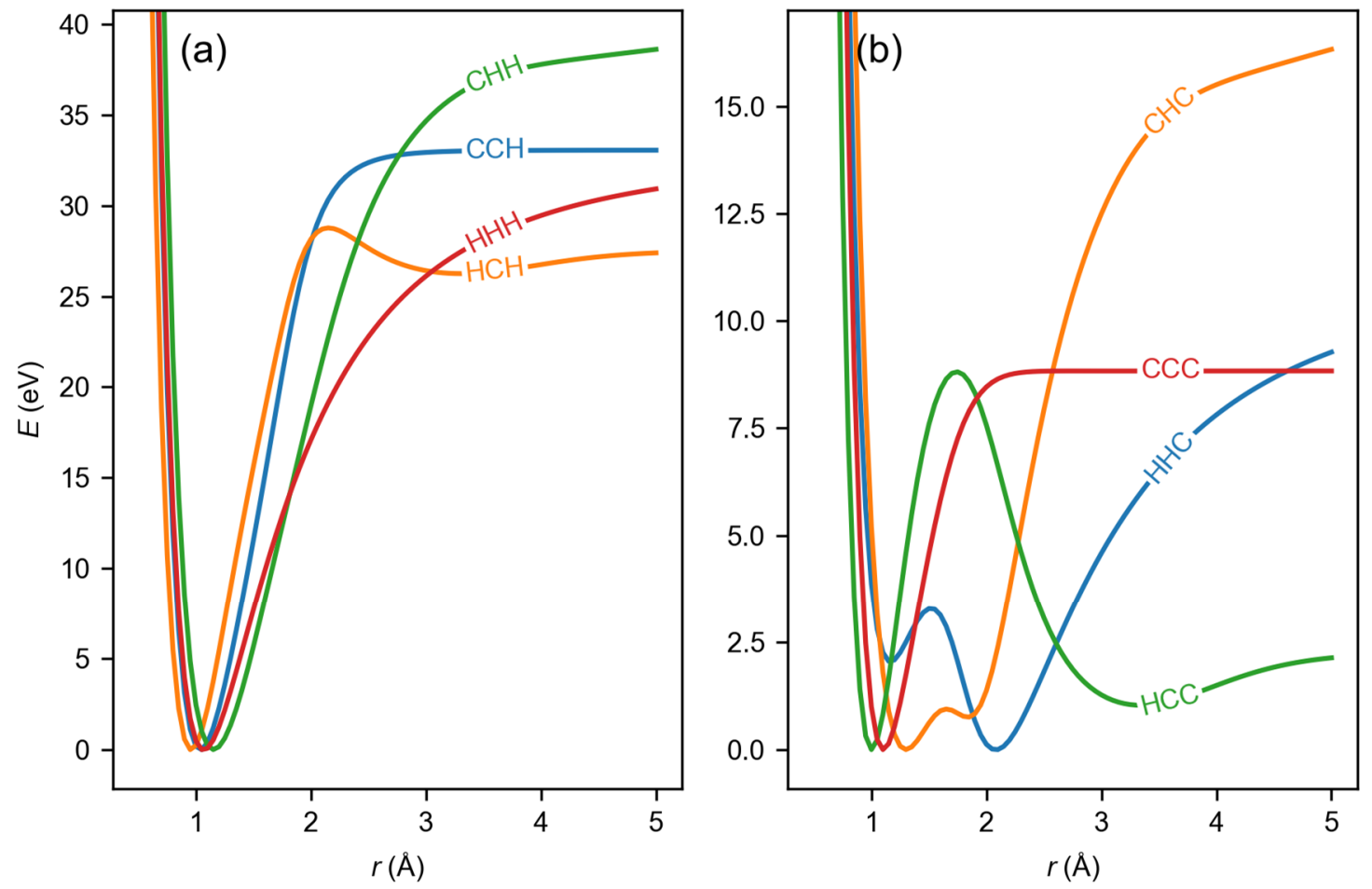

Figure S2. Dissociation curves of $\mathrm{N}_{2}$ using the DCC decomposition for RHF and CASSCF. (a) combinations with $E^{\mathrm{con}}$ from RHF. (b) combinations with $E^{\mathrm{con}}$ from CASSCF. 

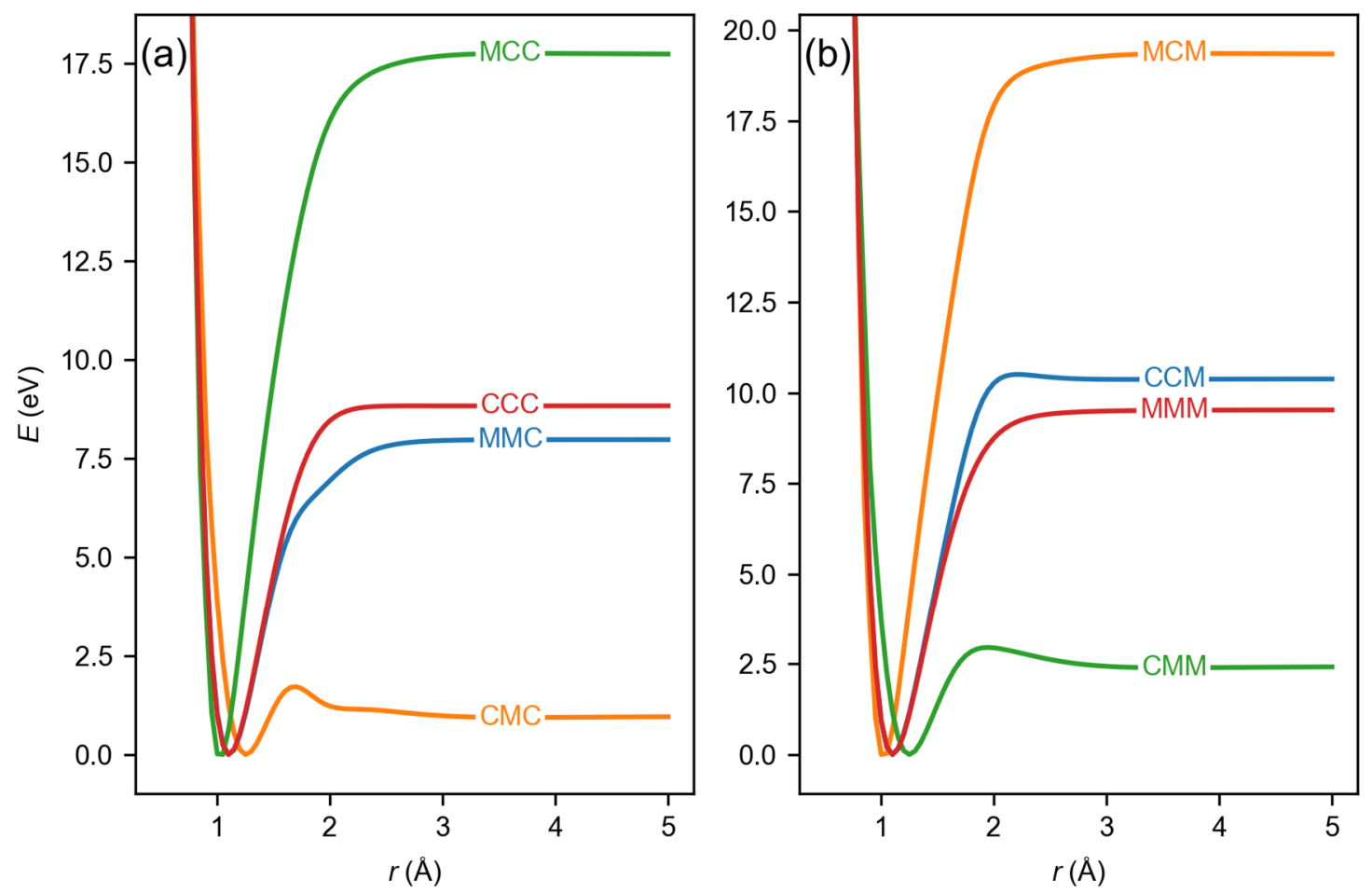

Figure S3. Dissociation curves of $\mathrm{N}_{2}$ using the DCC decomposition for CASSCF and MRCI. (a) combinations with $E^{\mathrm{con}}$ from CASSCF. (b) combinations with $E^{\mathrm{con}}$ from MRCI. 
Table S1 Absolute energies in hartrees of the DCC decomposition for $\mathrm{H}_{2}$.

\begin{tabular}{|c|c|c|c|c|c|c|c|c|c|c|c|c|c|}
\hline$r(\AA)$ & $E^{\text {nuc }}$ & $\begin{array}{c}E^{\mathrm{d}} \\
\mathrm{RHF}\end{array}$ & $\begin{array}{c}E^{\mathrm{d}} \\
\text { CASSCF }\end{array}$ & $\begin{array}{c}E^{\mathrm{d}} \\
\mathrm{MRCI}\end{array}$ & $\begin{array}{c}E^{\mathrm{dc}} \\
\mathrm{RHF}\end{array}$ & $\begin{array}{c}E^{\mathrm{dc}} \\
\text { CASSCF }\end{array}$ & $\begin{array}{c}E^{\mathrm{dc}} \\
\mathrm{MRCI}\end{array}$ & $\begin{array}{l}E^{\text {con }} \\
\text { RHF }\end{array}$ & $\begin{array}{c}E^{\text {con }} \\
\text { CASSCF }\end{array}$ & $\begin{array}{c}E^{\mathrm{con}} \\
\mathrm{MRCI}\end{array}$ & $\begin{array}{c}E^{\text {tot }} \\
\mathrm{RHF}\end{array}$ & $\begin{array}{c}E^{\text {tot }} \\
\text { CASSCF }\end{array}$ & $\begin{array}{c}E^{\text {tot }} \\
\text { MRCI }\end{array}$ \\
\hline 0.50 & 1.05835 & -1.27772 & -1.26351 & -1.24348 & 0.21441 & 0.21897 & 0.22583 & 0.00000 & -0.03164 & -0.08322 & -1.06331 & -1.07617 & -1.10087 \\
\hline 0.55 & 0.96214 & -1.25809 & -1.25766 & -1.24180 & 0.16270 & 0.18287 & 0.19264 & 0.00000 & -0.03447 & -0.08403 & -1.09539 & -1.10926 & -1.13319 \\
\hline 0.60 & 0.88196 & -1.21653 & -1.22942 & -1.21678 & 0.10112 & 0.13663 & 0.14830 & 0.00000 & -0.03757 & -0.08504 & -1.11541 & -1.13036 & -1.15352 \\
\hline 0.65 & 0.81412 & -1.16178 & -1.18789 & -1.17732 & 0.03495 & 0.08587 & 0.09828 & 0.00000 & -0.04094 & -0.08627 & -1.12684 & -1.14296 & -1.16532 \\
\hline 0.70 & 0.75597 & -1.10002 & -1.13924 & -1.12969 & -0.03206 & 0.03441 & 0.04644 & 0.00000 & -0.04461 & -0.08776 & -1.13208 & -1.14944 & -1.17101 \\
\hline 0.75 & 0.70557 & -1.03572 & -1.08768 & -1.07831 & -0.09710 & -0.01525 & -0.00447 & 0.00000 & -0.04861 & -0.08952 & -1.13282 & -1.15153 & -1.17230 \\
\hline 0.80 & 0.66147 & -0.97210 & -1.03603 & -1.02630 & -0.15819 & -0.06147 & -0.05253 & 0.00000 & -0.05294 & -0.09158 & -1.13029 & -1.15044 & -1.17041 \\
\hline 0.85 & 0.62256 & -0.91135 & -0.98618 & -0.97576 & -0.21403 & -0.10325 & -0.09651 & 0.00000 & -0.05764 & -0.09396 & -1.12538 & -1.14707 & -1.16622 \\
\hline 0.90 & 0.58797 & -0.85482 & -0.93938 & -0.92812 & -0.26391 & -0.13997 & -0.13561 & 0.00000 & -0.06272 & -0.09668 & -1.11873 & -1.14208 & -1.16041 \\
\hline 0.95 & 0.55703 & -0.80327 & -0.89647 & -0.88433 & -0.30757 & -0.17128 & -0.16938 & 0.00000 & -0.06820 & -0.09976 & -1.11083 & -1.13595 & -1.15347 \\
\hline 1.00 & 0.52918 & -0.75698 & -0.85791 & -0.84492 & -0.34509 & -0.19707 & -0.19762 & 0.00000 & -0.07408 & -0.10321 & -1.10206 & -1.12906 & -1.14576 \\
\hline 1.05 & 0.50398 & -0.71593 & -0.82392 & -0.81013 & -0.37676 & -0.21740 & -0.22038 & 0.00000 & -0.08038 & -0.10706 & -1.09269 & -1.12170 & -1.13757 \\
\hline 1.10 & 0.48107 & -0.67991 & -0.79442 & -0.77993 & -0.40303 & -0.23256 & -0.23786 & 0.00000 & -0.08710 & -0.11132 & -1.08293 & -1.11407 & -1.12912 \\
\hline 1.15 & 0.46015 & -0.64854 & -0.76919 & -0.75412 & -0.42441 & -0.24292 & -0.25044 & 0.00000 & -0.09424 & -0.11601 & -1.07295 & -1.10635 & -1.12058 \\
\hline 1.20 & 0.44098 & -0.62140 & -0.74790 & -0.73238 & -0.44147 & -0.24896 & -0.25855 & 0.00000 & -0.10180 & -0.12114 & -1.06287 & -1.09867 & -1.11207 \\
\hline 1.25 & 0.42334 & -0.59805 & -0.73016 & -0.71432 & -0.45473 & -0.25118 & -0.26266 & 0.00000 & -0.10978 & -0.12672 & -1.05278 & -1.09112 & -1.10370 \\
\hline 1.30 & 0.40706 & -0.57804 & -0.71555 & -0.69954 & -0.46473 & -0.25008 & -0.26325 & 0.00000 & -0.11815 & -0.13275 & -1.04276 & -1.08378 & -1.09555 \\
\hline 1.35 & 0.39198 & -0.56095 & -0.70369 & -0.68765 & -0.47192 & -0.24614 & -0.26079 & 0.00000 & -0.12688 & -0.13924 & -1.03287 & -1.07672 & -1.08768 \\
\hline 1.40 & 0.37798 & -0.54640 & -0.69420 & -0.67827 & -0.47674 & -0.23982 & -0.25571 & 0.00000 & -0.13595 & -0.14616 & -1.02314 & -1.06997 & -1.08014 \\
\hline 1.45 & 0.36495 & -0.53407 & -0.68673 & -0.67105 & -0.47954 & -0.23154 & -0.24842 & 0.00000 & -0.14529 & -0.15349 & -1.01361 & -1.06356 & -1.07296 \\
\hline 1.50 & 0.35278 & -0.52364 & -0.68100 & -0.66567 & -0.48066 & -0.22168 & -0.23930 & 0.00000 & -0.15485 & -0.16119 & -1.00430 & -1.05753 & -1.06617 \\
\hline 1.55 & 0.34140 & -0.51487 & -0.67671 & -0.66186 & -0.48036 & -0.21060 & -0.22870 & 0.00000 & -0.16456 & -0.16922 & -0.99524 & -1.05187 & -1.05978 \\
\hline 1.60 & 0.33074 & -0.50754 & -0.67362 & -0.65935 & -0.47887 & -0.19863 & -0.21695 & 0.00000 & -0.17435 & -0.17752 & -0.98642 & -1.04660 & -1.05381 \\
\hline 1.65 & 0.32071 & -0.50147 & -0.67154 & -0.65792 & -0.47638 & -0.18606 & -0.20434 & 0.00000 & -0.18412 & -0.18600 & -0.97785 & -1.04172 & -1.04826 \\
\hline 1.70 & 0.31128 & -0.49651 & -0.67026 & -0.65737 & -0.47304 & -0.17316 & -0.19116 & 0.00000 & -0.19380 & -0.19459 & -0.96955 & -1.03722 & -1.04313 \\
\hline 1.75 & 0.30239 & -0.49252 & -0.66964 & -0.65754 & -0.46899 & -0.16016 & -0.17767 & 0.00000 & -0.20330 & -0.20320 & -0.96151 & -1.03310 & -1.03840 \\
\hline 1.80 & 0.29399 & -0.48942 & -0.66954 & -0.65825 & -0.46431 & -0.14726 & -0.16408 & 0.00000 & -0.21254 & -0.21175 & -0.95373 & -1.02934 & -1.03408 \\
\hline 1.85 & 0.28604 & -0.48710 & -0.66983 & -0.65939 & -0.45911 & -0.13464 & -0.15062 & 0.00000 & -0.22145 & -0.22013 & -0.94621 & -1.02592 & -1.03014 \\
\hline 1.90 & 0.27851 & -0.48550 & -0.67042 & -0.66083 & -0.45344 & -0.12246 & -0.13746 & 0.00000 & -0.22996 & -0.22828 & -0.93895 & -1.02283 & -1.02657 \\
\hline
\end{tabular}




\begin{tabular}{|c|c|c|c|c|c|c|c|c|c|c|c|c|c|}
\hline 95 & 7 & 6 & 0 & 49 & -0.44738 & 31 & 5 & 0 & 1 & 11 & 93 & 5 & \\
\hline 2.00 & 26459 & -0.48420 & -0.67217 & -0.66426 & -0.44097 & -0.09980 & -0.11262 & 00000 & -0.24558 & -0.24357 & -0.92516 & -1.01756 & -1.02046 \\
\hline 2.05 & 58814 & -0.48437 & -0.67321 & -0.66611 & .43427 & -0.08949 & 10116 & 00000 & 25262 & -0.25060 & -0.91864 & -1.01533 & 1787 \\
\hline 2.10 & 0.25199 & -0.48501 & -0.67430 & -0.66796 & -0.42733 & -0.07991 & -0.09044 & .00000 & -0.25914 & -0.25716 & -0.91234 & -1.01335 & -1.01556 \\
\hline 2.20 & 0.24054 & -0.48749 & -0.67647 & -0.67150 & -0.41294 & -0.06302 & -0.07138 & 0.00000 & -0.27056 & -0.26882 & -0.90043 & -1.01004 & -1.01171 \\
\hline 2.25 & 0.23519 & -0.48922 & -0.67750 & -0.67314 & -0.40558 & -0.05569 & -0.06306 & 0.00000 & -0.27550 & -0.27391 & -0.89480 & -1.00868 & -1.01011 \\
\hline 2.40 & 49 & -0.4 & -0.68023 & -0.6 & -0.38 & & -0 . & 00 & & & 14 & -1.0 & -1.00642 \\
\hline 2.45 & 0.21599 & -0.49817 & -0.68099 & -0.67852 & -0.37613 & -0.03308 & -0.03729 & 0.00000 & -0.29063 & -0.28968 & -0.87430 & -1.00471 & -1.00549 \\
\hline 2.50 & & -0 & -0.68 & & -0.3 & & & & & & 65 & & -1 \\
\hline 2.55 & 0.20752 & -0.50327 & -0.68232 & -0.68048 & -0.36190 & -0.02520 & -0.02830 & 0.00000 & -0.29589 & -0.29519 & -0.86517 & -1.00341 & -1.00398 \\
\hline 2.60 & & & -0.6 & & & & & 00 & & & 86 & -1 & 37 \\
\hline 2.65 & 0.19969 & -0.50843 & -0.68338 & -0.68202 & -0.34828 & -0.01910 & -0.02136 & 0.00000 & -0.29995 & -0.29946 & -0.8 & -1.00 & -1.00284 \\
\hline 2.85 & 0.18568 & -0.51838 & -0.68485 & -0.68411 & -0.32326 & -0.01086 & -0.01204 & 0.00000 & -0.30544 & -0.30522 & -0.84164 & -1.00115 & -1.00137 \\
\hline 2.90 & 8247 & -0.52072 & -0.68511 & -0.68448 & -0.31750 & -0.00942 & -0.01043 & 0.00000 & -0.30640 & -0.30622 & -0.83822 & -1.00093 & -1.00112 \\
\hline 2.95 & 0.17938 & -0.52300 & -0.68534 & -0.68480 & -0.31193 & -0.00817 & -0.00902 & 0.00000 & -0.30723 & -0.30709 & -0.83493 & -1.00074 & -1.00091 \\
\hline 3.00 & 7639 & -0.52521 & -0.68555 & -0.68507 & -0.30656 & -0.00708 & -0.00781 & 0.00000 & -0.30795 & -0.30784 & -0.83177 & -1.00058 & -1.00073 \\
\hline 3.05 & 0.17350 & -0.52734 & -0.68572 & -0.68532 & -0.30138 & -0.00614 & -0.00676 & 0.00000 & -0.30858 & -0.30849 & -0.82872 & -1.00044 & -1.00057 \\
\hline 3.10 & 0.17070 & -0.52940 & -0.68588 & -0.68553 & -0.29639 & -0.00533 & -0.00585 & 0.00000 & -0.30912 & -0.30905 & -0.82579 & -1.00032 & -1.00043 \\
\hline 3.15 & 0.16799 & -0.53139 & -0.68602 & -0.68571 & -0.29158 & -0.00462 & -0.00507 & 0.00000 & -0.30958 & -0.30954 & -0.82297 & -1.00022 & -1.00032 \\
\hline 3.20 & 0.16537 & -0.53330 & -0.68614 & -0.68587 & -0.28695 & -0.00401 & -0.00439 & 0.00000 & -0.30999 & -0.30996 & -0.82026 & -1.00013 & -1.00022 \\
\hline 3.25 & 0.16282 & -0.53515 & -0.68625 & -0.68601 & -0.28250 & -0.00347 & -0.00380 & 0.00000 & -0.31034 & -0.31032 & -0.81765 & -1.00006 & -1.00013 \\
\hline 3.30 & 0.16036 & -0.53692 & -0.68634 & -0.68614 & -0.27822 & -0.00301 & -0.00329 & 0.00000 & -0.31064 & -0.31063 & -0.81514 & -0.99999 & -1.00006 \\
\hline 3.35 & 0.15796 & -0.53861 & -0.68642 & -0.68625 & -0.27410 & -0.00261 & -0.00285 & 0.00000 & -0.31090 & -0.31090 & -0.81272 & -0.99994 & -1.00000 \\
\hline
\end{tabular}




\begin{tabular}{|c|c|c|c|c|c|c|c|c|c|c|c|c|c|}
\hline 3.55 & 6 & & & 57 & & 48 & 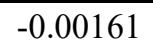 & 0 & 53 & 65 & 92 & 79 & \\
\hline 3.60 & 4699 & -0.54605 & -0.68672 & -0.68663 & .25587 & -0.00129 & -0.00140 & 0000 & -0.31176 & -0.31177 & -0.80192 & -0.99976 & -0.99980 \\
\hline 3.65 & 4498 & -0.54733 & -0.68676 & -0.68668 & 25267 & -0.00112 & .00121 & 0000 & 86 & -0.31188 & 000 & 974 & 977 \\
\hline 3.70 & 4302 & -0.54854 & -0.68680 & -0.68672 & -0.24960 & -0.00097 & -0.00105 & 00000 & -0.31195 & -0.31197 & -0.79815 & -0.99973 & -0.99975 \\
\hline 3.80 & 3926 & -0.5 & -0.68686 & -0.68680 & -0.24387 & -0.00074 & -0.00080 & 0000 & 10 & -0.31212 & -0.79465 & & -0.99972 \\
\hline 3.85 & 0.13745 & -0.55180 & -0.6 & -0.6 & -0.24119 & -0.00064 & -0.00069 & 00000 & -0.31216 & -0.31218 & -0.79299 & -0.99969 & -0.99970 \\
\hline 4.00 & & & -0.6 & & & & & & & & & & \\
\hline 4.05 & 66 & -0.5 & -0.6 & -0.6 & -0.2 & -0.0 & -0.0 & & -0 . & 34 & 697 & 66 & -0 \\
\hline 4.10 & & & & & & & & & & & & & \\
\hline 4.15 & 2751 & -0.55670 & -0.68699 & -0.68696 & -0.22757 & -0.00028 & -0.00 & 00 & -0.3 & -0.3 & -0 & -0.5 & -0.9 \\
\hline 4.20 & 9 & & & & & & & & & & & & \\
\hline 4.25 & 0.12451 & -0.55790 & -0.68701 & -0.68699 & -0.22387 & -0.00021 & -0.00023 & 0.00000 & -0.3 & -0.3 & -0.78177 & -0.9 & -0.99965 \\
\hline 4.45 & 0.11892 & -0.5 & -0.68704 & -0.6 & -0.21752 & -0.00012 & -0.00014 & 0.00000 & -0.31247 & -0.31248 & -0.77728 & -0.99963 & -0.99964 \\
\hline 4.50 & 0.11759 & -0.56013 & -0.68705 & -0.68703 & -0.21613 & -0.00011 & -0.00012 & 0.00000 & -0.31248 & -0.31249 & -0.77626 & -0.99963 & -0.99964 \\
\hline 4.55 & 0.11630 & -0.56046 & -0.68705 & -0.68703 & -0.21481 & -0.00009 & -0.00010 & 0.00000 & -0.31248 & -0.31249 & -0.77527 & -0.99963 & -0.99963 \\
\hline 4.60 & 0.11504 & -0.56075 & -0.68705 & -0.68704 & -0.21356 & -0.00008 & -0.00009 & 0.00000 & -0.31249 & -0.31250 & -0.77431 & -0.99963 & -0.99963 \\
\hline 4.65 & 0.11380 & -0.56102 & -0.68706 & -0.68705 & -0.21237 & -0.00007 & -0.00008 & 0.00000 & -0.31250 & -0.31251 & -0.77339 & -0.99963 & -0.99963 \\
\hline 4.70 & 0.11259 & -0.56126 & -0.68706 & -0.68705 & -0.21124 & -0.00006 & -0.00007 & 0.00000 & -0.31250 & -0.31251 & -0.77250 & -0.99963 & -0.99963 \\
\hline 4.75 & 0.11141 & -0.56147 & -0.68706 & -0.68705 & -0.21017 & -0.00005 & -0.00006 & 0.00000 & -0.31251 & -0.31251 & -0.77164 & -0.99962 & -0.99963 \\
\hline 4.80 & 0.11025 & -0.56166 & -0.68707 & -0.68706 & -0.20915 & -0.00005 & -0.00005 & 0.00000 & -0.31251 & -0.31252 & -0.77081 & -0.99962 & -0.99963 \\
\hline 4.85 & 0.10911 & -0.56182 & -0.68707 & -0.68706 & -0.20818 & -0.00004 & -0.00005 & 0.00000 & -0.31251 & -0.31252 & -0.77000 & -0.99962 & -0.99963 \\
\hline 4.90 & 0.10800 & -0.56197 & -0.68707 & -0.68706 & -0.20726 & -0.00004 & -0.00004 & 0.00000 & -0.31252 & -0.31252 & -0.76923 & -0.99962 & -0.99963 \\
\hline 4.95 & 0.10690 & -0.56210 & -0.68707 & -0.68706 & -0.20638 & -0.00003 & -0.00004 & 0.00000 & -0.31252 & -0.31252 & -0.76847 & -0.99962 & -0.99963 \\
\hline 5.00 & 0.10584 & -0.56221 & -0.68707 & -0.68707 & -0.20554 & -0.00003 & -0.00003 & 0.00000 & -0.31252 & -0.31253 & -0.76774 & -0.99962 & -0.99963 \\
\hline
\end{tabular}


Table S2 Absolute energies in hartrees of the DCC decomposition for $\mathrm{F}_{2}$.

\begin{tabular}{|c|c|c|c|c|c|c|c|c|c|c|c|c|c|}
\hline & & $E^{\mathrm{d}}$ & $E^{\mathrm{d}}$ & $E^{\mathrm{d}}$ & $E^{\mathrm{dc}}$ & $\overline{E^{\mathrm{dc}}}$ & $E^{\mathrm{dc}}$ & $E^{\text {con }}$ & $E^{\text {con }}$ & $E^{\text {con }}$ & $E^{\text {tot }}$ & $E^{\text {tot }}$ & $E^{\text {tot }}$ \\
\hline & & RHF & CASSCF & MRCI & RHF & CASSCF & MRCI & RHF & CASSCF & MRCI & RHF & CASSCF & MRCI \\
\hline 0.50 & 85.72671 & -174.66227 & -174.52155 & -174.09302 & -16.05249 & -16.18793 & -16.18240 & 0.00000 & -0.00995 & -0.91418 & -190.71475 & -190.71943 & -191.18960 \\
\hline 0.55 & 77.93337 & 93.24987 & 3.17891 & 2.11659 & 0.23912 & 0.17433 & -0.45196 & 00000 & -0.01157 & -0.92022 & -193.01074 & -193.01615 & -193.48876 \\
\hline 0.60 & 71.43892 & 99.95754 & 99.92693 & -199.07311 & 5.32191 & 5.29867 & 4.88181 & 0.00000 & -0.01375 & -0.92415 & -194.63564 & -194.64201 & -195.11546 \\
\hline 0.65 & 65.94362 & -202.01397 & -202.00814 & -201.48229 & 6.21696 & 6.22005 & 6.13111 & 0.00000 & -0.01648 & -0.92653 & -195.79701 & -195.80457 & -196.27771 \\
\hline 0.70 & 61.23336 & -202.29078 & -202.30098 & -202.02978 & 5.65723 & 5.67821 & 5.84316 & 0.00000 & -0.01977 & -0.92809 & -196.63355 & -196.64254 & -197.11471 \\
\hline 0.75 & 57.15114 & -201.93746 & -201.95875 & -201.84527 & 4.69795 & 4.73227 & 5.05368 & 0.00000 & -0.02367 & -0.92949 & -197.23951 & -197.25015 & -197.72108 \\
\hline 0.80 & 53.57919 & -201.40869 & -201.43842 & -201.40802 & 3.72877 & 3.77419 & 4.17710 & 0.00000 & -0.02823 & -0.93123 & -197.67991 & -197.69246 & -198.16216 \\
\hline 0.85 & 50.42748 & -200.87822 & -200.91510 & -200.92161 & 2.87788 & 2.93357 & 3.37162 & 0.00000 & -0.03353 & -0.93370 & -198.00034 & -198.01506 & -198.48369 \\
\hline 0.90 & 47.62595 & -200.40748 & -200.45093 & -200.47062 & 2.17428 & 2.24027 & 2.68959 & 0.00000 & -0.03972 & -0.93718 & -198.23319 & -198.25038 & -198.71821 \\
\hline 0.95 & 45.11932 & -200.01250 & -200.06225 & -200.08519 & 1.61074 & 1.68743 & 2.13798 & 0.00000 & -0.04697 & -0.94185 & -198.40176 & -198.42179 & -198.88906 \\
\hline 1.00 & 42.86335 & -199.69036 & -199.74608 & -199.74489 & 1.16741 & 1.25538 & 1.67928 & 0.00000 & -0.05555 & -0.94723 & -198.52295 & -198.54625 & -199.01284 \\
\hline 1.05 & 40.82224 & -199.43082 & -199.49201 & -199.49143 & 0.82177 & 0.92159 & 1.34342 & 0.00000 & -0.06574 & -0.95445 & -198.60906 & -198.63616 & -199.10246 \\
\hline 1.10 & 38.96669 & -199.22211 & -199.28790 & -199.28805 & 0.55303 & 0.66511 & 1.08452 & 0.00000 & -0.07782 & -0.96301 & -198.66908 & -198.70061 & -199.16654 \\
\hline 1.15 & 37.27248 & -199.05361 & -199.12269 & -199.12369 & 0.34399 & 0.46841 & 0.88496 & 0.00000 & -0.09198 & -0.97292 & -198.70961 & -198.74625 & -199.21165 \\
\hline 1.20 & 35.71946 & -198.91678 & -198.98741 & -198.98897 & 0.18125 & 0.31772 & 0.73056 & 0.00000 & -0.10830 & -0.98420 & -198.73553 & -198.77799 & -199.24261 \\
\hline 1.25 & 34.29068 & -198.80523 & -198.87528 & -198.87677 & 0.05478 & 56 & 0.61058 & 0.00000 & -0.12674 & -0.99683 & -198.75045 & -198.79947 & -199.26302 \\
\hline 1.30 & 32.97181 & -198.71427 & -198.78135 & -198.78207 & -0.04284 & 10 & 0.51730 & 0.00000 & -0.14715 & -1.01081 & -198.75712 & -198.81339 & -199.27558 \\
\hline 1.35 & 31.75063 & -198.64050 & -198.70203 & -198.70131 & -0.11711 & 0.04946 & 0.44504 & 0.00000 & -0.16925 & -1.02611 & -198.75 & -198.82182 & -199.28238 \\
\hline 1.40 & 30.61668 & -198.58131 & -198.63470 & -198.63196 & -0.17223 & 0.00109 & 0.38959 & 0.00000 & -0.19269 & -1.04263 & -198.75355 & -198.82631 & -199.28500 \\
\hline 1.45 & 29.56093 & -198.53465 & -198.57739 & -198.57214 & -0.21151 & -0.03362 & 0.34771 & 0.00000 & -0.21703 & -1.06026 & -198.74615 & -198.82804 & -199.28468 \\
\hline 1.50 & 28.57557 & -198.49881 & -198.52858 & -198.52043 & -0.23759 & -0.05754 & 0.31683 & 0.00000 & -0.24177 & -1.07878 & -198.73640 & -198.82790 & -199.28238 \\
\hline 1.55 & 27.65378 & -198.47233 & -198.48712 & -198.47570 & -0.25268 & -0.07304 & 0.29483 & 0.00000 & -0.26639 & -1.09795 & -198.72501 & -198.82655 & -199.27883 \\
\hline 1.60 & 26.78960 & -198.45388 & -198.45208 & -198.43708 & -0.25868 & -0.08202 & 0.27996 & 0.00000 & -0.29039 & -1.11745 & -198.71256 & -198.82449 & -199.27456 \\
\hline 1.65 & 25.97779 & -198.44218 & -198.42265 & -198.40381 & -0.25731 & -0.08611 & 0.27072 & 0.00000 & -0.31331 & -1.13690 & -198.69949 & -198.82207 & -199.26999 \\
\hline 1.70 & 25.21374 & -198.43599 & -198.39816 & -198.37527 & -0.25013 & -0.08660 & 0.26580 & 0.00000 & -0.33477 & -1.15594 & -198.68612 & -198.81953 & -199.26541 \\
\hline 1.75 & 24.49335 & -198.43411 & -198.37797 & -198.35089 & -0.23859 & -0.08459 & 0.26406 & 0.00000 & -0.35449 & -1.17420 & -198.67271 & -198.81705 & -199.26103 \\
\hline 1.80 & 23.81297 & -198.43542 & -198.36147 & -198.33015 & -0.22402 & -0.08095 & 0.26457 & 0.00000 & -0.37230 & -1.19137 & -198.65944 & -198.81472 & -199.25696 \\
\hline 1.85 & 23.16938 & -198.43890 & -198.34813 & -198.31260 & -0.20755 & -0.07637 & 0.26652 & 0.00000 & -0.38812 & -1.20720 & -198.64646 & -198.81262 & -199.25328 \\
\hline 1.90 & 22.55966 & -198.44371 & -198.33744 & -198.29781 & -0.19014 & -0.07135 & 0.26932 & 0.00000 & -0.40198 & -1.22153 & -198.63385 & -198.81076 & -199.25002 \\
\hline
\end{tabular}




\begin{tabular}{|c|c|c|c|c|c|c|c|c|c|c|c|c|c|}
\hline 1.95 & 21.98121 & -198.44920 & -198.32896 & -198.28543 & -0.17249 & -0.06624 & 0.27252 & 0.00000 & -0.41396 & -1.23428 & -198.62169 & -198.80916 & -199.24719 \\
\hline 2.00 & 21.43168 & -198.45486 & -198.32232 & -198.27513 & -0.15514 & -0.06126 & .27582 & 0.00000 & -0.42421 & -1.24545 & -198.61000 & -198.80779 & -199.24475 \\
\hline 2.05 & 20.90895 & -198.46036 & -198.31721 & -198.26663 & -0.13846 & -0.05653 & 27906 & 0.00000 & -0.43289 & -1.25510 & -198.59882 & -198.80664 & -199.24268 \\
\hline 2.10 & 20.41112 & -198.46547 & -198.31334 & -198.25969 & -0.12268 & -0.05215 & 0.28210 & 0.00000 & -0.44019 & -1.26334 & -198.58815 & -198.80568 & -199.24093 \\
\hline 2.15 & 19.93644 & -198.47004 & -198.31046 & -198.25406 & -0.10795 & -0.04813 & 0.28491 & 0.00000 & -0.44629 & -1.27031 & -198.57798 & -198.80488 & -199.23946 \\
\hline 2.20 & 19.48334 & -198.47399 & -198.30841 & -198.24955 & -0.09433 & -0.04446 & 0.28746 & 0.00000 & -0.45136 & -1.27614 & -198.56833 & -198.80423 & -199.23823 \\
\hline 2.25 & 19.05038 & -198.47730 & -198.30700 & -198.24599 & -0.08186 & -0.04114 & 0.28977 & 0.00000 & -0.45555 & -1.28100 & -198.55916 & -198.80369 & -199.23721 \\
\hline 2.30 & 18.63624 & -198.47995 & -198.30609 & -198.24319 & -0.07052 & -0.03816 & .29184 & 0.00000 & -0.45900 & -1.28502 & -198.55047 & -198.80325 & -199.23637 \\
\hline 2.35 & 18.23973 & -198.48195 & -198.30559 & -198.24104 & -0.06029 & -0.03547 & 0.29370 & 0.00000 & -0.46184 & -1.28833 & -198.54224 & -198.80290 & -199.23567 \\
\hline 2.40 & 17.85973 & -198.48331 & -198.30533 & -198.23940 & -0.05114 & -0.03311 & 35 & 0000 & -0.4 & -1.29104 & -198.5 & -198.80261 & -199 \\
\hline 2.45 & 17.49525 & -198.48405 & -198.30527 & -198.23817 & -0.04302 & -0.03103 & .29680 & 0.00000 & -0.46608 & -1.29326 & -198.52707 & -198.80238 & -199.23462 \\
\hline 2.50 & 17.14534 & -198.48420 & -198.30534 & -198.23725 & -0.03589 & -0.02921 & 0.29808 & 0.00000 & -0.46765 & -1.29507 & -198.52009 & -198.80220 & -199.23424 \\
\hline 2.55 & 16.80916 & -198.48380 & -198.30549 & -198.23657 & -0.02970 & -0.02764 & 0.29919 & 0.00000 & -0.46893 & -1.29654 & -198.51350 & -198.80206 & -199.23392 \\
\hline 2.60 & 16.48591 & -198.48286 & -198.30568 & -198.23608 & -0.02439 & -0.02630 & 0.30016 & 0.00000 & -0.46997 & 774 & -198.5 & -198.80195 & -199. \\
\hline 2.65 & 16.17485 & -198.48144 & -198.30587 & -198.23573 & -0.01992 & -0.02516 & 0.30098 & 0.00000 & -0.47083 & -1.29871 & -198.50136 & -198.80186 & -199.23345 \\
\hline 2.70 & 15.87532 & -198.47958 & -198.30606 & -198.23548 & -0.01621 & -0.02420 & 0.30169 & 0.00000 & -0.47153 & -1.29950 & -198.49579 & -198.80180 & -199.23329 \\
\hline 2.75 & 15.58667 & -198.47731 & -198.30624 & -198.23529 & -0.01321 & -0.02341 & 0.30228 & 0.00000 & -0.47210 & -1.30014 & -198.49052 & -198.80175 & -199.23315 \\
\hline 2.80 & 15.30834 & -198.47471 & -198.30638 & -198.23516 & -0.01084 & -0.02277 & 0.30277 & 0.00000 & -0.47257 & -1.30065 & -198.48555 & -198.80171 & -199.23304 \\
\hline 2.85 & 15.03977 & -198.47181 & -198.30649 & -198.23506 & -0.00904 & -0.02225 & 0.30318 & 0.00000 & -0.47295 & -1.30107 & -198.48085 & -198.80169 & -199.23295 \\
\hline 2.90 & 14.78047 & -198.46868 & -198.30657 & -198.23498 & -0.00773 & -0.02184 & 0.30351 & 0.00000 & -0.47327 & -1.30142 & -198.47641 & -198.80168 & -199.23289 \\
\hline 2.95 & 14.52995 & -198.46537 & -198.30662 & -198.23492 & -0.00685 & -0.02152 & 0.30378 & 0.00000 & -0.47353 & -1.30169 & -198.47222 & -198.80167 & -199.23283 \\
\hline 3.00 & 14.28778 & -198.46195 & -198.30665 & -198.23487 & -0.00631 & -0.02127 & 0.30400 & 0.00000 & -0.47374 & -1.30192 & -198.46826 & -198.80166 & -199.23279 \\
\hline 3.05 & 14.05356 & -198.45846 & -198.30665 & -198.23482 & -0.00607 & -0.02109 & 0.30417 & 0.00000 & -0.47392 & -1.30210 & -198.46453 & -198.80166 & -199.23275 \\
\hline 3.10 & 13.82689 & -198.45496 & -198.30664 & -198.23478 & -0.00605 & -0.02096 & 0.30430 & 0.00000 & -0.47407 & -1.30226 & -198.46101 & -198.80167 & -199.23273 \\
\hline 3.15 & 13.60741 & -198.45149 & -198.30661 & -198.23474 & -0.00619 & -0.02087 & 0.30441 & 0.00000 & -0.47419 & -1.30238 & -198.45769 & -198.80167 & -199.23271 \\
\hline 3.20 & 13.39480 & -198.44809 & -198.30657 & -198.23470 & -0.00646 & -0.02081 & 0.30449 & 0.00000 & -0.47430 & -1.30248 & -198.45455 & -198.80168 & -199.23269 \\
\hline 3.25 & 13.18872 & -198.44480 & -198.30652 & -198.23467 & -0.00680 & -0.02077 & 0.30456 & 0.00000 & -0.47438 & -1.30257 & -198.45159 & -198.80168 & -199.23268 \\
\hline 3.30 & 12.98890 & -198.44163 & -198.30647 & -198.23465 & -0.00718 & -0.02076 & 0.30462 & 0.00000 & -0.47446 & -1.30264 & -198.44880 & -198.80169 & -199.23267 \\
\hline 3.35 & 12.79503 & -198.43860 & -198.30642 & -198.23461 & -0.00757 & -0.02075 & 0.30465 & 0.00000 & -0.47452 & -1.30270 & -198.44617 & -198.80169 & -199.23267 \\
\hline 3.40 & 12.60687 & -198.43575 & -198.30637 & -198.23460 & -0.00794 & -0.02076 & 0.30469 & 0.00000 & -0.47457 & -1.30275 & -198.44369 & -198.80170 & -199.23266 \\
\hline 3.45 & 12.42416 & -198.43306 & -198.30632 & -198.23459 & -0.00828 & -0.02077 & 0.30473 & 0.00000 & -0.47462 & -1.30280 & -198.44134 & -198.80171 & -199.23266 \\
\hline 3.50 & 12.24667 & -198.43055 & -198.30627 & -198.23459 & -0.00858 & -0.02078 & 0.30477 & 0.00000 & -0.47466 & -1.30283 & -198.43913 & -198.80171 & -199.23266 \\
\hline
\end{tabular}




\begin{tabular}{|c|c|c|c|c|c|c|c|c|c|c|c|c|c|}
\hline 3.55 & .07418 & 98.42822 & -198.30623 & 198.23460 & -0.00002 & -0.02080 & 30481 & 0.00000 & -0.47469 & -1.30286 & -198.43704 & -198.80172 & -19 \\
\hline 3.60 & 11.90649 & -198.42606 & -198.30619 & -198.23462 & -0.00900 & -0.02082 & 30485 & 0.00000 & -0.47472 & -1.30288 & -198.43506 & -198.80173 & -199.23266 \\
\hline 3.65 & 11.74338 & 98.42408 & -198.30616 & -198.23464 & -0.00912 & -0.02083 & 0488 & 0.00000 & -0.47474 & -1.30290 & 3320 & -198.80173 & 3266 \\
\hline 3.70 & 11.58469 & -198.42226 & -198.30613 & -198.23466 & -0.00917 & -0.02085 & 30493 & 0.00000 & -0.47476 & -1.30292 & -198.43143 & -198.80174 & -199.23266 \\
\hline 3.75 & 11.43023 & -198.42060 & -198.30610 & -198.23469 & -0.00916 & -0.02086 & 30496 & 0.00000 & -0.47478 & -1.30294 & -198.42976 & -198.80175 & -199.23266 \\
\hline 3.80 & 11.27983 & -198.41910 & -198.30608 & -198.23472 & -0.00909 & -0.02088 & 30501 & 0.00000 & -0.47480 & -1.30295 & -198.42818 & -198.80175 & -199.23266 \\
\hline 3.85 & 11.13334 & -198.41773 & -198.30606 & -198.23476 & -0.00896 & -0.02089 & 30505 & 0.00000 & -0.47481 & -1.30296 & -198.42669 & -198.80176 & -199.23266 \\
\hline 3.90 & 10.99060 & -198.41650 & -198 & -198. & -0.00 & -0.0 & 09 & 0000 & & -1.3 & -19 & -198 & 267 \\
\hline 3.95 & 10.85148 & -198.41539 & -198.30603 & -198.23484 & -0.00854 & -0.02091 & 30514 & 0.00000 & -0.47483 & -1.30297 & -198.42392 & -198.80177 & -199.23267 \\
\hline 4.00 & 10.71584 & -198. & -198 & -198 & -0.0 & & & 00 & & & 64 & & 267 \\
\hline 4.05 & 10.58354 & -198.41350 & -198.30601 & -198.23492 & -0.00793 & -0.02093 & 30523 & 0.00000 & -0.47484 & -1.30298 & -198.4 & -198.80178 & -199 \\
\hline 4.10 & 10.45 & -198 & -198 & -198 & & & & & & & & & \\
\hline 4.15 & 10.32852 & -198.41199 & -198.30599 & -198.23501 & -0.00718 & -0.02094 & 0.30532 & 0.00000 & -0.47485 & -1.30299 & -198.41917 & -198 & -199 \\
\hline 4.20 & 10.20556 & -198. & -198 & -198. & & & & & & & & 179 & \\
\hline 4.25 & 10.08550 & -198.41081 & -198.30598 & -198.23511 & -0.00631 & -0.02095 & 0.30542 & 0.00000 & -0.47486 & -1.30300 & -198 & -198.8 & -199 \\
\hline 4.30 & 9.96822 & -198.41031 & -198.30598 & -198.23516 & -0.00585 & -0.02096 & 30547 & 0.00000 & -0.47487 & -1.30300 & -198.41616 & -198.80180 & -199 \\
\hline 4.35 & 9.85364 & -198.40988 & -198.30597 & -198.23521 & -0.00536 & -0.02096 & 0.30551 & 0.00000 & -0.47487 & -1.30300 & -198.41524 & -198.80180 & -199.23269 \\
\hline 4.40 & 9.74167 & -198.40950 & -198.30597 & -198.23525 & -0.00486 & -0.02096 & 556 & 0.00000 & -0.47487 & -1.30300 & -198.41436 & -198.80181 & -199.23270 \\
\hline 4.45 & 9.63221 & -198.40917 & -198.30597 & -198.23530 & -0.00435 & -0.02097 & 0.30560 & 0.00000 & -0.47487 & -1.30300 & -198.41352 & -198.80181 & -199.23270 \\
\hline 4.50 & 9.52519 & -198.40887 & -198.30597 & -198.23534 & -0.00384 & -0.02097 & .30565 & 0.00000 & -0.47488 & -1.30300 & -198.41271 & -198.80181 & -199.23270 \\
\hline 4.55 & 9.42052 & -198.40862 & -198.30597 & -198.23539 & -0.00331 & -0.02097 & 0.30569 & 0.00000 & -0.47488 & -1.30300 & -198.41193 & -198.80182 & -199.23270 \\
\hline 4.60 & 9.31812 & -198.40840 & -198.30597 & -198.23544 & -0.00278 & -0.02097 & 0.30573 & 0.00000 & -0.47488 & -1.30300 & -198.41118 & -198.80182 & -199.23271 \\
\hline 4.65 & 9.21793 & -198.40820 & -198.30596 & -198.23548 & -0.00225 & -0.02098 & 0.30578 & 0.00000 & -0.47488 & -1.30300 & -198.41045 & -198.80182 & -199.23271 \\
\hline 4.70 & 9.11986 & -198.40804 & -198.30596 & -198.23553 & -0.00171 & -0.02098 & 0.30582 & 0.00000 & -0.47488 & -1.30300 & -198.40975 & -198.80182 & -199.23271 \\
\hline 4.75 & 9.02386 & -198.40789 & -198.30596 & -198.23557 & -0.00118 & -0.02098 & 0.30587 & 0.00000 & -0.47488 & -1.30301 & -198.40907 & -198.80183 & -199.23271 \\
\hline 4.80 & 8.92987 & -198.40776 & -198.30596 & -198.23561 & -0.00065 & -0.02098 & 0.30591 & 0.00000 & -0.47488 & -1.30301 & -198.40841 & -198.80183 & -199.23271 \\
\hline 4.85 & 8.83780 & -198.40765 & -198.30596 & -198.23565 & -0.00012 & -0.02099 & 0.30595 & 0.00000 & -0.47489 & -1.30301 & -198.40778 & -198.80183 & -199.23271 \\
\hline 4.90 & 8.74762 & -198.40756 & -198.30596 & -198.23570 & 0.00040 & -0.02099 & 0.30599 & 0.00000 & -0.47489 & -1.30301 & -198.40716 & -198.80183 & -199.23272 \\
\hline 4.95 & 8.65926 & -198.40747 & -198.30596 & -198.23574 & 0.00092 & -0.02099 & 0.30603 & 0.00000 & -0.47489 & -1.30301 & -198.40656 & -198.80183 & -199.23272 \\
\hline 5.00 & 8.57267 & -198.40740 & -198.30596 & -198.23578 & 0.00143 & -0.02099 & 0.30607 & 0.00000 & -0.47489 & -1.30301 & -198.40597 & -198.80184 & -199.23272 \\
\hline
\end{tabular}


Table S3 Absolute energies in hartrees of the DCC decomposition for $\mathrm{N}_{2}$.

\begin{tabular}{|c|c|c|c|c|c|c|c|c|c|c|c|c|c|}
\hline & & $E^{\mathrm{d}}$ & $E^{\mathrm{d}}$ & $E^{\mathrm{d}}$ & $E^{\mathrm{dc}}$ & $E^{\mathrm{dc}}$ & $\overline{E^{\mathrm{dc}}}$ & $E^{\text {con }}$ & $E^{\text {con }}$ & $E^{\text {con }}$ & $E^{\text {tot }}$ & $E^{\text {tot }}$ & $E^{\text {tot }}$ \\
\hline & & RHF & CASSCF & MRCI & RHF & CASSCF & MRCI & RHF & CASSCF & MRCI & RHF & ASSCF & MRCI \\
\hline 0.50 & 51.85937 & -97.50799 & -95.64010 & -93.63896 & -5.94865 & -7.76349 & -9.48651 & 0.00000 & -0.10141 & -0.66404 & -103.45664 & -103.50500 & -103.78951 \\
\hline 0.55 & 47.14488 & -105.64723 & -104.50040 & -103.38260 & 0.55396 & -0.53540 & -1.37870 & 0.00000 & -0.10960 & -0.66493 & -105.09326 & -105.14540 & -105.42623 \\
\hline 0.60 & 43.21614 & -109.64838 & -108.86087 & -108.26078 & 3.36283 & 2.63791 & 2.30965 & 0.00000 & -0.11902 & -0.66898 & -106.28554 & -106.34198 & -106.62012 \\
\hline 0.65 & 39.89182 & -111.40740 & -110.85433 & -110.59378 & 4.26151 & 3.77686 & 3.78626 & 0.00000 & -0.12982 & -0.67602 & -107.14589 & -107.20729 & -107.48354 \\
\hline 0.70 & 37.04240 & -111.98700 & -111.60503 & -111.57519 & 4.22597 & 3.91896 & 4.15771 & 0.00000 & -0.14200 & -0.68545 & -107.76103 & -107.82807 & -108.10293 \\
\hline 0.75 & 34.57291 & -111.96159 & -111.70937 & -111.83282 & 3.76559 & 3.59555 & 3.98647 & 0.00000 & -0.15553 & -0.69668 & -108.19600 & -108.26935 & -108.54303 \\
\hline 0.80 & 32.41210 & -111.64586 & -111.49437 & -111.71174 & 3.14701 & 3.08559 & 3.56941 & 0.00000 & -0.17040 & -0.70941 & -108.49885 & -108.57919 & -108.85174 \\
\hline 0.85 & 30.50551 & -111.20982 & -111.13795 & -111.40388 & 2.50497 & 2.53169 & 3.06308 & 0.00000 & -0.18658 & -0.72348 & -108.70485 & -108.79284 & -109.06428 \\
\hline 0.90 & 28.81076 & -110.74293 & -110.73507 & -110.98380 & 1.90306 & 2.00296 & 2.51797 & 0.00000 & -0.20407 & -0.74107 & -108.83988 & -108.93619 & -109.20690 \\
\hline 0.95 & 27.29440 & -110.29012 & -110.33450 & -110.58755 & 1.36720 & 1.52912 & 2.04748 & 0.00000 & -0.22287 & -0.75775 & -108.92293 & -109.02825 & -109.29782 \\
\hline 1.00 & 25.92968 & -109.87183 & -109.95936 & -110.19631 & 0.90382 & 1.11931 & 1.62067 & 0.00000 & -0.24297 & -0.77590 & -108.96802 & -109.08301 & -109.35154 \\
\hline 1.05 & 24.69494 & -109.49558 & -109.61916 & -109.82885 & 0.51010 & 0.77269 & 1.24599 & 0.00000 & -0.26438 & -0.79558 & -108.98548 & -109.11084 & -109.37844 \\
\hline 1.10 & 23.57244 & -109.16287 & -109.31678 & -109.49504 & 0.17986 & 0.48450 & 0.92566 & 0.00000 & -0.28713 & -0.81686 & -108.98301 & -109.11940 & -109.38624 \\
\hline 1.15 & 22.54755 & -108.87291 & -109.05228 & -109.19951 & -0.09339 & 0.24913 & 0.65863 & 0.00000 & -0.31126 & -0.83979 & -108.96630 & -109.11441 & -109.38067 \\
\hline 1.20 & 21.60807 & -108.62420 & -108.82449 & -108.94358 & -0.31544 & 0.06121 & 0.44197 & 0.00000 & -0.33685 & -0.86438 & -108.93964 & -109.10013 & -109.36600 \\
\hline 1.25 & 20.74375 & -108.41475 & -108.63146 & -108.72617 & -0.49148 & -0.08432 & 0.27141 & 0.00000 & -0.36398 & -0.89065 & -108.90623 & -109.07976 & -109.3 \\
\hline 1.30 & 19.94591 & -108.24186 & -108.47046 & -108.54449 & -0.62659 & -0.19241 & 0.14187 & 0.00000 & -0.39281 & -0.91862 & -108.86845 & -109.05568 & -109.32124 \\
\hline 1.35 & 19.20717 & -108.10202 & -108.33805 & -108.39466 & -0.72605 & -0.26810 & 0.04772 & 0.00000 & -0.42351 & -0.94831 & -108.82807 & -109.02966 & -109.29525 \\
\hline 1.40 & 18.52120 & -107.99106 & -108.23030 & -108.27210 & -0.79535 & -0.31632 & -0.01679 & 0.00000 & -0.45637 & -0.97980 & -108.78641 & -109.00300 & -109.26869 \\
\hline 1.45 & 17.88254 & -107.90453 & -108.14316 & -108.17207 & -0.83988 & -0.34179 & -0.05724 & 0.00000 & -0.49169 & -1.01314 & -108.74441 & -108.97664 & -109.24246 \\
\hline 1.50 & 17.28646 & -107.83806 & -108.07273 & -108.09012 & -0.86469 & -0.34870 & -0.07861 & 0.00000 & -0.52985 & -1.04846 & -108.70275 & -108.95128 & -109.21719 \\
\hline 1.55 & 16.72883 & -107.78768 & -108.01556 & -108.02222 & -0.87420 & -0.34068 & -0.08525 & 0.00000 & -0.57118 & -1.08585 & -108.66188 & -108.92742 & -109.19332 \\
\hline 1.60 & 16.20605 & -107.74999 & -107.96872 & -107.96507 & -0.87215 & -0.32080 & -0.08073 & 0.00000 & -0.61589 & -1.12535 & -108.62214 & -108.90541 & -109.17115 \\
\hline 1.65 & 15.71496 & -107.72212 & -107.92983 & -107.91601 & -0.86160 & -0.29180 & -0.06803 & 0.00000 & -0.66387 & -1.16684 & -108.58373 & -108.88550 & -109.15089 \\
\hline 1.70 & 15.25275 & -107.70195 & -107.89701 & -107.87307 & -0.84482 & -0.25636 & -0.04956 & 0.00000 & -0.71449 & -1.21001 & -108.54678 & -108.86787 & -109.13265 \\
\hline 1.75 & 14.81696 & -107.68775 & -107.86875 & -107.83472 & -0.82360 & -0.21727 & -0.02758 & 0.00000 & -0.76657 & -1.25419 & -108.51136 & -108.85259 & -109.11648 \\
\hline 1.80 & 14.40538 & -107.67827 & -107.84385 & -107.79991 & -0.79924 & -0.17742 & -0.00402 & 0.00000 & -0.81839 & -1.29847 & -108.47750 & -108.83966 & -109.10239 \\
\hline 1.85 & 14.01605 & -107.67254 & -107.82136 & -107.76787 & -0.77268 & -0.13951 & 0.01925 & 0.00000 & -0.86811 & -1.34169 & -108.44522 & -108.82898 & -109.09032 \\
\hline 1.90 & 13.64720 & -107.66985 & -107.80057 & -107.73814 & -0.74463 & -0.10572 & 0.04069 & 0.00000 & -0.91408 & -1.38270 & -108.41448 & -108.82038 & -109.08015 \\
\hline
\end{tabular}




\begin{tabular}{|c|c|c|c|c|c|c|c|c|c|c|c|c|c|}
\hline 1.95 & .29727 & -107.66965 & -107.78106 & -107.71045 & .71561 & -0.07736 & 5925 & 0.00000 & -0.95520 & -1.42050 & -108.38526 & -108.81361 & -109 \\
\hline 2.00 & 12.96484 & -107.67149 & -107.76258 & -107.68470 & -0.68603 & -0.05489 & 0.07435 & 0.00000 & -0.99094 & -1.45445 & -108.35752 & -108.80840 & -109.06480 \\
\hline 2.05 & 12.64863 & -107.67500 & -107.74513 & -107.66090 & -0.65620 & -0.03804 & 85992 & 0.00000 & -1.02131 & -1.48424 & -108.33120 & -108.80448 & -109.05922 \\
\hline 2.10 & 12.34747 & -107.67984 & -107.72882 & -107.63908 & -0.62639 & -0.02606 & 0.09423 & 0.00000 & -1.04669 & -1.50990 & -108.30624 & -108.80158 & -109.05475 \\
\hline 2.15 & 12.06032 & -107.68573 & -107.71381 & -107.61931 & -0.59686 & -0.01802 & 0.09978 & 0.00000 & -1.06764 & -1.53166 & -108.28258 & -108.79947 & -109.05120 \\
\hline 2.20 & 11.78622 & -107.69238 & -107.70021 & -107.60161 & -0.56779 & -0.01295 & 0.10313 & 0.00000 & -1.08479 & -1.54990 & -108.26017 & -108.79795 & -109.04838 \\
\hline 2.25 & 11.52430 & -107.69956 & -107.68809 & -107.58594 & -0.53939 & -0.01002 & 0.10486 & 0.00000 & -1.09877 & -1.56507 & -108.23894 & -108.79688 & -109.04615 \\
\hline 2.30 & 11.27378 & -107.70704 & -107.67748 & -107.57228 & -0.5 & -0.00853 & 10549 & 0.00000 & -1.11013 & -1.57760 & -108.21884 & -108.7 & -109.04439 \\
\hline 2.35 & 11.03391 & -107.71464 & -107.66833 & -107.56041 & -0.48515 & -0.00792 & 0.10534 & 0.00000 & -1.11938 & -1.58791 & -108.19979 & -108.79563 & -109.04299 \\
\hline 2.40 & 10.80403 & -107.72220 & -107.66054 & -107.55025 & -0.45956 & -0.00784 & 76 & 0.00000 & -1.12691 & 639 & -108 & -108 & -109. \\
\hline 2.45 & 10.58354 & -107.72958 & -107.65402 & -107.54164 & -0.43509 & -0.00798 & 0.10399 & 0.00000 & -1.13306 & -1.60334 & -108.16467 & -108.79507 & -109.04099 \\
\hline 2.50 & 10.37187 & -107 & -107 & $-10^{7}$ & -0.41 & -0.00 & 20 & 0.0 & -1 & -1 & -108 & -108 & -10 \\
\hline 2.55 & 10.16850 & -107.74344 & -107.64424 & -107.52840 & -0.38969 & -0.00837 & 0.10244 & 0.00000 & -1.14223 & -1.61373 & -108.13313 & -108.79484 & -109.03969 \\
\hline 2.60 & 9.97296 & -107 & -107 & -107 & -0 & -0.00 & 80 & 00 & -1 & -1 & -10 & -10 & -10 \\
\hline 2.65 & 9.78479 & -107.75574 & -107.63793 & -107.51937 & -0.34904 & -0.00839 & 0.10130 & 0.00000 & -1.14846 & -1.62075 & -108.10478 & -108.79477 & -109.03882 \\
\hline 2.70 & 9.60359 & -107.76123 & -107.63576 & -107.51608 & & -0.00822 & 5 & 0.00000 & 79 & 37 & -108.0 & -108 & -109. \\
\hline 2.75 & 9.42898 & -107.76627 & -107.63411 & -107.51344 & -0.31300 & -0.00793 & 0.10075 & 0.00000 & -1.15273 & -1.62552 & -108.07927 & -108.79476 & -109.03822 \\
\hline 2.80 & 9.26060 & -107.77086 & -107.63288 & -107.51135 & -0.29661 & -0.00754 & 0.10067 & 0.00000 & -1.15434 & -1.62730 & -108.06747 & -108.79476 & -109.03798 \\
\hline 2.85 & 9.09813 & -107.77501 & -107.63200 & -107.50971 & -0.28127 & -0.00708 & 0.10070 & 0.00000 & -1.15569 & -1.62877 & -108.05627 & -108.79477 & -109.03778 \\
\hline 2.90 & 8.94127 & -107.77871 & -107.63140 & -107.50844 & -0.26691 & -0.00657 & 0.10082 & 0.00000 & -1.15681 & -1.62998 & -108.04562 & -108.79477 & -109.03760 \\
\hline 2.95 & 8.78972 & -107.78199 & -107.63101 & -107.50748 & -0.25351 & -0.00602 & 0.10102 & 0.00000 & -1.15775 & -1.63099 & -108.03550 & -108.79478 & -109.03745 \\
\hline 3.00 & 8.64323 & -107.78486 & -107.63078 & -107.50676 & -0.24101 & -0.00546 & 0.10126 & 0.00000 & -1.15854 & -1.63182 & -108.02587 & -108.79478 & -109.03731 \\
\hline 3.05 & 8.50154 & -107.78732 & -107.63069 & -107.50619 & -0.22939 & -0.00489 & 0.10150 & 0.00000 & -1.15920 & -1.63251 & -108.01670 & -108.79479 & -109.03720 \\
\hline 3.10 & 8.36441 & -107.78938 & -107.63068 & -107.50582 & -0.21860 & -0.00435 & 0.10181 & 0.00000 & -1.15976 & -1.63308 & -108.00797 & -108.79479 & -109.03709 \\
\hline 3.15 & 8.23165 & -107.79106 & -107.63074 & -107.50557 & -0.20860 & -0.00382 & 0.10212 & 0.00000 & -1.16023 & -1.63355 & -107.99966 & -108.79479 & -109.03700 \\
\hline 3.20 & 8.10303 & -107.79236 & -107.63084 & -107.50541 & -0.19937 & -0.00333 & 0.10244 & 0.00000 & -1.16062 & -1.63394 & -107.99173 & -108.79479 & -109.03692 \\
\hline 3.25 & 7.97836 & -107.79330 & -107.63097 & -107.50532 & -0.19086 & -0.00287 & 0.10275 & 0.00000 & -1.16095 & -1.63427 & -107.98416 & -108.79479 & -109.03685 \\
\hline 3.30 & 7.85748 & -107.79390 & -107.63111 & -107.50529 & -0.18304 & -0.00244 & 0.10304 & 0.00000 & -1.16124 & -1.63453 & -107.97694 & -108.79479 & -109.03678 \\
\hline 3.35 & 7.74020 & -107.79416 & -107.63125 & -107.50529 & -0.17588 & -0.00207 & 0.10332 & 0.00000 & -1.16147 & -1.63475 & -107.97004 & -108.79479 & -109.03672 \\
\hline 3.40 & 7.62638 & -107.79411 & -107.63139 & -107.50531 & -0.16934 & -0.00172 & 0.10358 & 0.00000 & -1.16167 & -1.63494 & -107.96345 & -108.79479 & -109.03667 \\
\hline 3.45 & 7.51585 & -107.79377 & -107.63152 & -107.50535 & -0.16338 & -0.00142 & 0.10382 & 0.00000 & -1.16184 & -1.63509 & -107.95715 & -108.79479 & -109.03663 \\
\hline 3.50 & 7.40848 & -107.79315 & -107.63163 & -107.50540 & -0.15797 & -0.00116 & 0.10403 & 0.00000 & -1.16199 & -1.63521 & -107.95112 & -108.79479 & -109.03658 \\
\hline
\end{tabular}




\begin{tabular}{|c|c|c|c|c|c|c|c|c|c|c|c|c|c|}
\hline 3.55 & 7.30414 & -107.79229 & -107.63174 & 107.50545 & -0.15306 & -0.00094 & 0422 & 0.00000 & -1.16211 & -1.63532 & -107.94535 & -108.79478 & -109 \\
\hline 3.60 & 7.20269 & -107.79122 & -107.63182 & -107.50551 & -0.14862 & -0.00075 & 10440 & 0.00000 & -1.16222 & -1.63541 & -107.93983 & -108.79478 & -109.03652 \\
\hline 3.65 & 7.10402 & -107.78994 & -107.63189 & -107.50556 & -0.14460 & -0.00058 & 10455 & 0.00000 & -1.16231 & -1.63548 & -107.93455 & -108.79478 & 3649 \\
\hline 3.70 & 7.00802 & -107.78851 & -107.63195 & -107.50560 & -0.14097 & -0.00045 & 10468 & 0.00000 & -1.16238 & -1.63554 & -107.92948 & -108.79478 & -109.03646 \\
\hline 3.75 & 6.91458 & -107.78694 & -107.63199 & -107.50564 & -0.13768 & -0.00034 & 10480 & 0.00000 & -1.16245 & -1.63559 & -107.92462 & -108.79478 & -109.03643 \\
\hline 3.80 & 6.82360 & -107.78527 & -107.63202 & -107.50568 & -0.13470 & -0.00025 & 0490 & 0.00000 & -1.16251 & -1.63564 & -107.91997 & -108.79478 & -109.03641 \\
\hline 3.85 & 6.73498 & -107.78351 & -107.63204 & -107.50571 & -0.13199 & -0.00018 & 10499 & 0.00000 & -1.16256 & -1.63567 & -107.91551 & -108.79477 & -109.03639 \\
\hline 3.90 & 6.64864 & -107.78171 & -107 & -107.50573 & -0.12952 & -0.00012 & 506 & 00 & -1.1 & & -107.9 & -108 & -109 \\
\hline 3.95 & 6.56448 & -107.77987 & -107.63205 & -107.50575 & -0.12724 & -0.00008 & 10513 & 0.00000 & -1.16264 & -1.63573 & -107.90712 & -108.79477 & -109.03636 \\
\hline 4.00 & 6.4 & -107 & $-10^{-}$ & -10 & & & & & & & & & \\
\hline 4.05 & 6.40239 & -107.77621 & -107.63203 & -107.50578 & -0.12318 & -0.00002 & 10523 & 0.00000 & -1.16271 & -1.6 & -107.89939 & -108.79476 & -109.03633 \\
\hline 4.10 & & -107 & $-10^{\prime}$ & -10 & & & & & & & & & \\
\hline 4.15 & 6.24812 & -107.77266 & -107.63200 & -107.50580 & -0.11960 & 0.00000 & 0.10531 & 0.00000 & -1.16276 & -1.63581 & -107.89226 & -108.7 & -109.03630 \\
\hline 4.20 & & -107. & -107. & -10 & -0.11794 & & & & -1.16278 & & & & \\
\hline 4.25 & 6.10110 & -107.76934 & -107.63196 & -107.50581 & -0.11634 & 0.00001 & 0.10537 & 0.00000 & -1.16280 & -1.63584 & -107.88568 & -108 & -109.03628 \\
\hline 4.30 & 6.03016 & -107.76778 & -107.63194 & -107.50582 & -0.11479 & 0.00001 & 10540 & 0.00000 & -1.16282 & -1.63585 & -107.88257 & -108.79475 & -109.03627 \\
\hline 4.35 & 5.96085 & -107.76631 & -107.63192 & -107.50583 & -0.11328 & 0.00001 & 0.10542 & 0.00000 & -1.16283 & -1.63586 & -107.87959 & -108.79474 & -109.03626 \\
\hline 4.40 & 5.89311 & -107.76491 & -107.63190 & -107 & -0.11180 & 0.00000 & 10545 & 0.00000 & -1.16285 & -1.63586 & -107.87672 & -108.79474 & -109.03625 \\
\hline 4.45 & 5.82690 & -107.76360 & -107.63188 & -107.50585 & -0.11035 & 0.00000 & 0.10547 & 0.00000 & -1.16286 & -1.63587 & -107.87395 & -108.79474 & -109.03625 \\
\hline 4.50 & 5.76215 & -107.76237 & -107.63186 & -107.50585 & -0.10891 & -0.00001 & 0.10549 & 0.00000 & -1.16287 & -1.63588 & -107.87128 & -108.79474 & -109.03624 \\
\hline 4.55 & 5.69883 & -107.76123 & -107.63185 & -107.50587 & -0.10748 & -0.00001 & 0.10551 & 0.00000 & -1.16288 & -1.63588 & -107.86871 & -108.79473 & -109.03623 \\
\hline 4.60 & 5.63689 & -107.76017 & -107.63183 & -107.50588 & -0.10607 & -0.00001 & 0.10554 & 0.00000 & -1.16288 & -1.63588 & -107.86624 & -108.79473 & -109.03623 \\
\hline 4.65 & 5.57628 & -107.75919 & -107.63182 & -107.50589 & -0.10466 & -0.00002 & 0.10556 & 0.00000 & -1.16289 & -1.63589 & -107.86385 & -108.79473 & -109.03622 \\
\hline 4.70 & 5.51695 & -107.75829 & -107.63181 & -107.50591 & -0.10325 & -0.00002 & 0.10558 & 0.00000 & -1.16290 & -1.63589 & -107.86154 & -108.79473 & -109.03622 \\
\hline 4.75 & 5.45888 & -107.75746 & -107.63180 & -107.50592 & -0.10185 & -0.00003 & 0.10560 & 0.00000 & -1.16290 & -1.63589 & -107.85931 & -108.79473 & -109.03621 \\
\hline 4.80 & 5.40202 & -107.75670 & -107.63179 & -107.50594 & -0.10045 & -0.00003 & 0.10562 & 0.00000 & -1.16290 & -1.63589 & -107.85715 & -108.79472 & -109.03621 \\
\hline 4.85 & 5.34633 & -107.75601 & -107.63178 & -107.50596 & -0.09906 & -0.00003 & 0.10565 & 0.00000 & -1.16291 & -1.63590 & -107.85507 & -108.79472 & -109.03621 \\
\hline 4.90 & 5.29177 & -107.75538 & -107.63178 & -107.50597 & -0.09767 & -0.00003 & 0.10567 & 0.00000 & -1.16291 & -1.63590 & -107.85305 & -108.79472 & -109.03620 \\
\hline 4.95 & 5.23832 & -107.75481 & -107.63177 & -107.50599 & -0.09629 & -0.00004 & 0.10569 & 0.00000 & -1.16291 & -1.63590 & -107.85110 & -108.79472 & -109.03620 \\
\hline 5.00 & 5.18594 & -107.75430 & -107.63177 & -107.50601 & -0.09491 & -0.00004 & 0.10571 & 0.00000 & -1.16292 & -1.63590 & -107.84921 & -108.79472 & -109.03620 \\
\hline
\end{tabular}


Table S4 Absolute energies in hartrees of the $\mathrm{CN}$ decomposition for $\mathrm{H}_{2}$.

\begin{tabular}{|c|c|c|c|c|c|c|c|}
\hline$r(\AA)$ & $E^{\text {nuc }}$ & $\begin{array}{c}E^{\mathrm{cl}} \\
\mathrm{RHF} \\
\end{array}$ & $\begin{array}{c}E^{\mathrm{cl}} \\
\mathrm{CASSCF}\end{array}$ & $\begin{array}{c}E^{\mathrm{cl}} \\
\mathrm{MRCI}\end{array}$ & $\begin{array}{l}E^{\text {noncl }} \\
\text { RHF }\end{array}$ & $\begin{array}{c}E^{\text {noncl }} \\
\text { CASSCF }\end{array}$ & $\begin{array}{c}E^{\text {noncl }} \\
\text { MRCI }\end{array}$ \\
\hline 0.50 & 1.05835 & -0.30442 & -0.28952 & -0.27184 & -0.75888 & -0.78666 & -0.82903 \\
\hline 0.55 & 0.96214 & -0.35996 & -0.34384 & -0.32689 & -0.73543 & -0.76542 & -0.80631 \\
\hline 0.60 & 0.88196 & -0.40199 & -0.38458 & -0.36839 & -0.71342 & -0.74579 & -0.78513 \\
\hline 0.65 & 0.81412 & -0.43407 & -0.41528 & -0.39989 & -0.69276 & -0.72768 & -0.76543 \\
\hline 0.70 & 0.75597 & -0.45871 & -0.43844 & -0.42387 & -0.67337 & -0.71101 & -0.74714 \\
\hline 0.75 & 0.70557 & -0.47769 & -0.45583 & -0.44212 & -0.65514 & -0.69570 & -0.73018 \\
\hline 0.80 & 0.66147 & -0.49231 & -0.46876 & -0.45592 & -0.63798 & -0.68168 & -0.71449 \\
\hline 0.85 & 0.62256 & -0.50355 & -0.47819 & -0.46623 & -0.62183 & -0.66888 & -0.70000 \\
\hline 0.90 & 0.58797 & -0.51213 & -0.48483 & -0.47377 & -0.60660 & -0.65724 & -0.68664 \\
\hline 0.95 & 0.55703 & -0.51861 & -0.48925 & -0.47910 & -0.59222 & -0.64670 & -0.67437 \\
\hline 1.00 & 0.52918 & -0.52342 & -0.49186 & -0.48263 & -0.57864 & -0.63720 & -0.66313 \\
\hline 1.05 & 0.50398 & -0.52689 & -0.49300 & -0.48470 & -0.56580 & -0.62870 & -0.65287 \\
\hline 1.10 & 0.48107 & -0.52929 & -0.49294 & -0.48557 & -0.55365 & -0.62113 & -0.64355 \\
\hline 1.15 & 0.46015 & -0.53082 & -0.49188 & -0.48545 & -0.54214 & -0.61447 & -0.63513 \\
\hline 1.20 & 0.44098 & -0.53165 & -0.49000 & -0.48448 & -0.53122 & -0.60867 & -0.62758 \\
\hline 1.25 & 0.42334 & -0.53192 & -0.48742 & -0.48282 & -0.52086 & -0.60370 & -0.62088 \\
\hline 1.30 & 0.40706 & -0.53173 & -0.48428 & -0.48056 & -0.51103 & -0.59950 & -0.61499 \\
\hline 1.35 & 0.39198 & -0.53118 & -0.48066 & -0.47779 & -0.50168 & -0.59606 & -0.60989 \\
\hline 1.40 & 0.37798 & -0.53034 & -0.47665 & -0.47458 & -0.49280 & -0.59332 & -0.60555 \\
\hline 1.45 & 0.36495 & -0.52927 & -0.47232 & -0.47101 & -0.48434 & -0.59125 & -0.60195 \\
\hline 1.50 & 0.35278 & -0.52801 & -0.46774 & -0.46713 & -0.47629 & -0.58979 & -0.59904 \\
\hline 1.55 & 0.34140 & -0.52661 & -0.46297 & -0.46298 & -0.46862 & -0.58890 & -0.59680 \\
\hline 1.60 & 0.33074 & -0.52510 & -0.45807 & -0.45864 & -0.46131 & -0.58854 & -0.59518 \\
\hline 1.65 & 0.32071 & -0.52351 & -0.45309 & -0.45413 & -0.45434 & -0.58864 & -0.59413 \\
\hline 1.70 & 0.31128 & -0.52186 & -0.44808 & -0.44952 & -0.44770 & -0.58915 & -0.59361 \\
\hline 1.75 & 0.30239 & -0.52016 & -0.44309 & -0.44484 & -0.44135 & -0.59001 & -0.59356 \\
\hline 1.80 & 0.29399 & -0.51844 & -0.43816 & -0.44015 & -0.43529 & -0.59117 & -0.59393 \\
\hline 1.85 & 0.28604 & -0.51670 & -0.43334 & -0.43549 & -0.42951 & -0.59258 & -0.59465 \\
\hline 1.90 & 0.27851 & -0.51496 & -0.42866 & -0.43091 & -0.42398 & -0.59417 & -0.59566 \\
\hline 1.95 & 0.27137 & -0.51323 & -0.42415 & -0.42643 & -0.41870 & -0.59590 & -0.59692 \\
\hline 2.00 & 0.26459 & -0.51151 & -0.41984 & -0.42210 & -0.41366 & -0.59771 & -0.59835 \\
\hline 2.05 & 0.25814 & -0.50980 & -0.41576 & -0.41795 & -0.40884 & -0.59957 & -0.59992 \\
\hline 2.10 & 0.25199 & -0.50811 & -0.41190 & -0.41400 & -0.40423 & -0.60145 & -0.60156 \\
\hline 2.15 & 0.24613 & -0.50645 & -0.40829 & -0.41027 & -0.39983 & -0.60330 & -0.60324 \\
\hline 2.20 & 0.24054 & -0.50481 & -0.40494 & -0.40678 & -0.39562 & -0.60510 & -0.60493 \\
\hline 2.25 & 0.23519 & -0.50319 & -0.40184 & -0.40353 & -0.39161 & -0.60684 & -0.60659 \\
\hline 2.30 & 0.23008 & -0.50161 & -0.39898 & -0.40052 & -0.38778 & -0.60850 & -0.60819 \\
\hline 2.35 & 0.22518 & -0.50004 & -0.39637 & -0.39776 & -0.38412 & -0.61006 & -0.60973 \\
\hline 2.40 & 0.22049 & -0.49851 & -0.39399 & -0.39523 & -0.38063 & -0.61152 & -0.61119 \\
\hline 2.45 & 0.21599 & -0.49699 & -0.39183 & -0.39293 & -0.37731 & -0.61288 & -0.61256 \\
\hline 2.50 & 0.21167 & -0.49551 & -0.38988 & -0.39085 & -0.37414 & -0.61413 & -0.61383 \\
\hline 2.55 & 0.20752 & -0.49404 & -0.38812 & -0.38898 & -0.37113 & -0.61528 & -0.61500 \\
\hline
\end{tabular}




\begin{tabular}{|c|c|c|c|c|c|c|c|}
\hline 2.60 & 0.20353 & -0.49261 & -0.38655 & -0.38729 & -0.36826 & -0.61633 & -0.61608 \\
\hline 2.65 & 0.19969 & -0.49119 & -0.38514 & -0.38578 & -0.36552 & -0.61729 & -0.61706 \\
\hline 2.70 & 0.19599 & -0.48980 & -0.38388 & -0.38444 & -0.36292 & -0.61816 & -0.61795 \\
\hline 2.75 & 0.19243 & -0.48844 & -0.38275 & -0.38324 & -0.36045 & -0.61894 & -0.61876 \\
\hline 2.80 & 0.18899 & -0.48710 & -0.38176 & -0.38218 & -0.35809 & -0.61964 & -0.61949 \\
\hline 2.85 & 0.18568 & -0.48579 & -0.38088 & -0.38123 & -0.35585 & -0.62027 & -0.62014 \\
\hline 2.90 & 0.18247 & -0.48450 & -0.38010 & -0.38040 & -0.35372 & -0.62083 & -0.62072 \\
\hline 2.95 & 0.17938 & -0.48324 & -0.37941 & -0.37967 & -0.35169 & -0.62133 & -0.62124 \\
\hline 3.00 & 0.17639 & -0.48200 & -0.37880 & -0.37902 & -0.34977 & -0.62178 & -0.62170 \\
\hline 3.05 & 0.17350 & -0.48078 & -0.37827 & -0.37846 & -0.34794 & -0.62217 & -0.62211 \\
\hline 3.10 & 0.17070 & -0.47960 & -0.37780 & -0.37796 & -0.34620 & -0.62253 & -0.62248 \\
\hline 3.15 & 0.16799 & -0.47843 & -0.37739 & -0.37752 & -0.34454 & -0.62283 & -0.62280 \\
\hline 3.20 & 0.16537 & -0.47729 & -0.37703 & -0.37714 & -0.34297 & -0.62311 & -0.62308 \\
\hline 3.25 & 0.16282 & -0.47618 & -0.37671 & -0.37681 & -0.34147 & -0.62335 & -0.62333 \\
\hline 3.30 & 0.16036 & -0.47509 & -0.37643 & -0.37652 & -0.34004 & -0.62356 & -0.62354 \\
\hline 3.35 & 0.15796 & -0.47403 & -0.37619 & -0.37626 & -0.33869 & -0.62375 & -0.62373 \\
\hline 3.40 & 0.15564 & -0.47299 & -0.37598 & -0.37604 & -0.33740 & -0.62391 & -0.62390 \\
\hline 3.45 & 0.15338 & -0.47197 & -0.37580 & -0.37584 & -0.33618 & -0.62405 & -0.62406 \\
\hline 3.50 & 0.15119 & -0.47098 & -0.37564 & -0.37568 & -0.33501 & -0.62418 & -0.62418 \\
\hline 3.55 & 0.14906 & -0.47001 & -0.37550 & -0.37553 & -0.33391 & -0.62429 & -0.62429 \\
\hline 3.60 & 0.14699 & -0.46907 & -0.37538 & -0.37540 & -0.33285 & -0.62438 & -0.62439 \\
\hline 3.65 & 0.14498 & -0.46815 & -0.37527 & -0.37529 & -0.33184 & -0.62447 & -0.62448 \\
\hline 3.70 & 0.14302 & -0.46726 & -0.37518 & -0.37520 & -0.33088 & -0.62454 & -0.62455 \\
\hline 3.75 & 0.14111 & -0.46639 & -0.37510 & -0.37512 & -0.32997 & -0.62461 & -0.62462 \\
\hline 3.80 & 0.13926 & -0.46555 & -0.37503 & -0.37504 & -0.32909 & -0.62466 & -0.62467 \\
\hline 3.85 & 0.13745 & -0.46473 & -0.37497 & -0.37498 & -0.32826 & -0.62471 & -0.62472 \\
\hline 3.90 & 0.13569 & -0.46394 & -0.37492 & -0.37493 & -0.32746 & -0.62475 & -0.62476 \\
\hline 3.95 & 0.13397 & -0.46317 & -0.37488 & -0.37488 & -0.32669 & -0.62479 & -0.62480 \\
\hline 4.00 & 0.13229 & -0.46243 & -0.37484 & -0.37484 & -0.32596 & -0.62483 & -0.62484 \\
\hline 4.05 & 0.13066 & -0.46171 & -0.37480 & -0.37480 & -0.32526 & -0.62485 & -0.62486 \\
\hline 4.10 & 0.12907 & -0.46101 & -0.37477 & -0.37477 & -0.32458 & -0.62488 & -0.62489 \\
\hline 4.15 & 0.12751 & -0.46034 & -0.37474 & -0.37474 & -0.32393 & -0.62490 & -0.62491 \\
\hline 4.20 & 0.12599 & -0.45970 & -0.37472 & -0.37472 & -0.32330 & -0.62492 & -0.62493 \\
\hline 4.25 & 0.12451 & -0.45908 & -0.37470 & -0.37470 & -0.32269 & -0.62494 & -0.62495 \\
\hline 4.30 & 0.12306 & -0.45848 & -0.37468 & -0.37468 & -0.32211 & -0.62495 & -0.62496 \\
\hline 4.35 & 0.12165 & -0.45791 & -0.37467 & -0.37467 & -0.32154 & -0.62497 & -0.62498 \\
\hline 4.40 & 0.12027 & -0.45735 & -0.37465 & -0.37465 & -0.32099 & -0.62498 & -0.62499 \\
\hline 4.45 & 0.11892 & -0.45683 & -0.37464 & -0.37464 & -0.32046 & -0.62499 & -0.62500 \\
\hline 4.50 & 0.11759 & -0.45632 & -0.37463 & -0.37463 & -0.31994 & -0.62500 & -0.62501 \\
\hline 4.55 & 0.11630 & -0.45584 & -0.37462 & -0.37462 & -0.31943 & -0.62501 & -0.62501 \\
\hline 4.60 & 0.11504 & -0.45538 & -0.37461 & -0.37461 & -0.31893 & -0.62501 & -0.62502 \\
\hline 4.65 & 0.11380 & -0.45494 & -0.37461 & -0.37460 & -0.31845 & -0.62502 & -0.62503 \\
\hline 4.70 & 0.11259 & -0.45452 & -0.37460 & -0.37460 & -0.31798 & -0.62503 & -0.62503 \\
\hline 4.75 & 0.11141 & -0.45412 & -0.37459 & -0.37459 & -0.31752 & -0.62503 & -0.62504 \\
\hline 4.80 & 0.11025 & -0.45375 & -0.37459 & -0.37459 & -0.31706 & -0.62504 & -0.62504 \\
\hline
\end{tabular}


S-15

\begin{tabular}{llllllll}
\hline 4.85 & 0.10911 & -0.45339 & -0.37458 & -0.37458 & -0.31662 & -0.62504 & -0.62504 \\
4.90 & 0.10800 & -0.45305 & -0.37458 & -0.37458 & -0.31618 & -0.62504 & -0.62505 \\
4.95 & 0.10690 & -0.45273 & -0.37458 & -0.37458 & -0.31575 & -0.62505 & -0.62505 \\
5.00 & 0.10584 & -0.45242 & -0.37457 & -0.37457 & -0.31532 & -0.62505 & -0.62505 \\
\hline
\end{tabular}


Table S5 Absolute energies in hartrees of the $\mathrm{CN}$ decomposition for $\mathrm{F}_{2}$.

\begin{tabular}{|c|c|c|c|c|c|c|c|}
\hline$r(\AA)$ & $E^{\text {nuc }}$ & $\begin{array}{c}E^{\mathrm{cl}} \\
\mathrm{RHF}\end{array}$ & $\begin{array}{c}E^{\mathrm{cl}} \\
\mathrm{CASSCF}\end{array}$ & $\begin{array}{c}E^{\mathrm{cl}} \\
\mathrm{MRCI}\end{array}$ & $\begin{array}{l}E^{\text {noncl }} \\
\text { RHF }\end{array}$ & $\begin{array}{c}E^{\text {noncl }} \\
\text { CASSCF }\end{array}$ & $\begin{array}{c}E^{\text {noncl }} \\
\text { MRCI }\end{array}$ \\
\hline 0.50 & 85.72671 & -168.88874 & -168.88403 & -168.53235 & -21.82601 & -21.83540 & -22.65724 \\
\hline 0.55 & 77.93337 & -171.39012 & -171.38458 & -171.04024 & -21.62062 & -21.63157 & -22.44852 \\
\hline 0.60 & 71.43892 & -173.20434 & -173.19773 & -172.86097 & -21.43130 & -21.44428 & -22.25449 \\
\hline 0.65 & 65.94362 & -174.54001 & -174.53212 & -174.20217 & -21.25700 & -21.27245 & -22.07554 \\
\hline 0.70 & 61.23336 & -175.53652 & -175.52713 & -175.20295 & -21.09703 & -21.11541 & -21.91175 \\
\hline 0.75 & 57.15114 & -176.28857 & -176.27744 & -175.95809 & -20.95094 & -20.97271 & -21.76300 \\
\hline 0.80 & 53.57919 & -176.86169 & -176.84860 & -176.53330 & -20.81822 & -20.84386 & -21.62886 \\
\hline 0.85 & 50.42748 & -177.30214 & -177.28681 & -176.97501 & -20.69821 & -20.72825 & -21.50869 \\
\hline 0.90 & 47.62595 & -177.64313 & -177.62525 & -177.31654 & -20.59006 & -20.62513 & -21.40167 \\
\hline 0.95 & 45.11932 & -177.90891 & -177.88810 & -177.58224 & -20.49286 & -20.53369 & -21.30682 \\
\hline 1.00 & 42.86335 & -178.11733 & -178.09314 & -177.79149 & -20.40562 & -20.45310 & -21.22135 \\
\hline 1.05 & 40.82224 & -178.28168 & -178.25359 & -177.95426 & -20.32738 & -20.38257 & -21.14819 \\
\hline 1.10 & 38.96669 & -178.41185 & -178.37929 & -178.08256 & -20.25723 & -20.32132 & -21.08398 \\
\hline 1.15 & 37.27248 & -178.51526 & -178.47767 & -178.18375 & -20.19435 & -20.26858 & -21.02791 \\
\hline 1.20 & 35.71946 & -178.59753 & -178.55437 & -178.26340 & -20.13800 & -20.22363 & -20.97921 \\
\hline 1.25 & 34.29068 & -178.66291 & -178.61371 & -178.32577 & -20.08754 & -20.18576 & -20.93725 \\
\hline 1.30 & 32.97181 & -178.71472 & -178.65909 & -178.37415 & -20.04240 & -20.15430 & -20.90144 \\
\hline 1.35 & 31.75063 & -178.75557 & -178.69322 & -178.41116 & -20.00204 & -20.12860 & -20.87122 \\
\hline 1.40 & 30.61668 & -178.78756 & -178.71829 & -178.43890 & -19.96599 & -20.10802 & -20.84610 \\
\hline 1.45 & 29.56093 & -178.81235 & -178.73612 & -178.45910 & -19.93380 & -20.09192 & -20.82559 \\
\hline 1.50 & 28.57557 & -178.83132 & -178.74821 & -178.47318 & -19.90507 & -20.07969 & -20.80920 \\
\hline 1.55 & 27.65378 & -178.84559 & -178.75581 & -178.48235 & -19.87942 & -20.07074 & -20.79648 \\
\hline 1.60 & 26.78960 & -178.85605 & -178.75997 & -178.48763 & -19.85651 & -20.06452 & -20.78693 \\
\hline 1.65 & 25.97779 & -178.86346 & -178.76158 & -178.48989 & -19.83603 & -20.06050 & -20.78011 \\
\hline 1.70 & 25.21374 & -178.86842 & -178.76133 & -178.48987 & -19.81770 & -20.05820 & -20.77554 \\
\hline 1.75 & 24.49335 & -178.87142 & -178.75983 & -178.48822 & -19.80128 & -20.05722 & -20.77280 \\
\hline 1.80 & 23.81297 & -178.87288 & -178.75752 & -178.48546 & -19.78657 & -20.05721 & -20.77150 \\
\hline 1.85 & 23.16938 & -178.87310 & -178.75476 & -178.48201 & -19.77336 & -20.05787 & -20.77127 \\
\hline 1.90 & 22.55966 & -178.87236 & -178.75180 & -178.47821 & -19.76149 & -20.05896 & -20.77181 \\
\hline 1.95 & 21.98121 & -178.87087 & -178.74884 & -178.47432 & -19.75081 & -20.06032 & -20.77287 \\
\hline 2.00 & 21.43168 & -178.86881 & -178.74599 & -178.47051 & -19.74119 & -20.06180 & -20.77424 \\
\hline 2.05 & 20.90895 & -178.86631 & -178.74332 & -178.46690 & -19.73250 & -20.06332 & -20.77578 \\
\hline 2.10 & 20.41112 & -178.86350 & -178.74089 & -178.46357 & -19.72465 & -20.06479 & -20.77735 \\
\hline 2.15 & 19.93644 & -178.86045 & -178.73871 & -178.46056 & -19.71753 & -20.06617 & -20.77890 \\
\hline 2.20 & 19.48334 & -178.85726 & -178.73678 & -178.45788 & -19.71107 & -20.06745 & -20.78036 \\
\hline 2.25 & 19.05038 & -178.85397 & -178.73508 & -178.45551 & -19.70519 & -20.06861 & -20.78170 \\
\hline 2.30 & 18.63624 & -178.85063 & -178.73360 & -178.45345 & -19.69984 & -20.06965 & -20.78292 \\
\hline 2.35 & 18.23973 & -178.84728 & -178.73232 & -178.45167 & -19.69496 & -20.07058 & -20.78401 \\
\hline 2.40 & 17.85973 & -178.84395 & -178.73123 & -178.45013 & -19.69050 & -20.07139 & -20.78496 \\
\hline 2.45 & 17.49525 & -178.84064 & -178.73030 & -178.44883 & -19.68642 & -20.07209 & -20.78580 \\
\hline 2.50 & 17.14534 & -178.83739 & -178.72951 & -178.44771 & -19.68270 & -20.07270 & -20.78652 \\
\hline 2.55 & 16.80916 & -178.83420 & -178.72884 & -178.44677 & -19.67929 & -20.07322 & -20.78715 \\
\hline
\end{tabular}




\begin{tabular}{|c|c|c|c|c|c|c|c|}
\hline 2.60 & 16.48591 & -178.83108 & -178.72827 & -178.44598 & -19.67618 & -20.07367 & -20.78769 \\
\hline 2.65 & 16.17485 & -178.82803 & -178.72780 & -178.44531 & -19.67333 & -20.07406 & -20.78815 \\
\hline 2.70 & 15.87532 & -178.82506 & -178.72740 & -178.44474 & -19.67073 & -20.07439 & -20.78854 \\
\hline 2.75 & 15.58667 & -178.82217 & -178.72707 & -178.44427 & -19.66835 & -20.07468 & -20.78888 \\
\hline 2.80 & 15.30834 & -178.81937 & -178.72679 & -178.44387 & -19.66618 & -20.07493 & -20.78917 \\
\hline 2.85 & 15.03977 & -178.81666 & -178.72655 & -178.44354 & -19.66419 & -20.07514 & -20.78942 \\
\hline 2.90 & 14.78047 & -178.81404 & -178.72636 & -178.44326 & -19.66237 & -20.07532 & -20.78963 \\
\hline 2.95 & 14.52995 & -178.81152 & -178.72619 & -178.44302 & -19.66070 & -20.07548 & -20.78981 \\
\hline 3.00 & 14.28778 & -178.80910 & -178.72605 & -178.44282 & -19.65917 & -20.07561 & -20.78997 \\
\hline 3.05 & 14.05356 & -178.80678 & -178.72593 & -178.44265 & -19.65776 & -20.07573 & -20.79011 \\
\hline 3.10 & 13.82689 & -178.80456 & -178.72583 & -178.44250 & -19.65645 & -20.07583 & -20.79023 \\
\hline 3.15 & 13.60741 & -178.80245 & -178.72574 & -178.44238 & -19.65524 & -20.07593 & -20.79033 \\
\hline 3.20 & 13.39480 & -178.80044 & -178.72567 & -178.44227 & -19.65411 & -20.07600 & -20.79042 \\
\hline 3.25 & 13.18872 & -178.79854 & -178.72561 & -178.44218 & -19.65305 & -20.07607 & -20.79050 \\
\hline 3.30 & 12.98890 & -178.79675 & -178.72555 & -178.44210 & -19.65205 & -20.07613 & -20.79057 \\
\hline 3.35 & 12.79503 & -178.79507 & -178.72551 & -178.44204 & -19.65110 & -20.07619 & -20.79063 \\
\hline 3.40 & 12.60687 & -178.79350 & -178.72547 & -178.44198 & -19.65019 & -20.07623 & -20.79068 \\
\hline 3.45 & 12.42416 & -178.79203 & -178.72544 & -178.44194 & -19.64931 & -20.07627 & -20.79072 \\
\hline 3.50 & 12.24667 & -178.79066 & -178.72541 & -178.44190 & -19.64847 & -20.07630 & -20.79076 \\
\hline 3.55 & 12.07418 & -178.78939 & -178.72539 & -178.44187 & -19.64764 & -20.07633 & -20.79079 \\
\hline 3.60 & 11.90649 & -178.78822 & -178.72537 & -178.44184 & -19.64684 & -20.07635 & -20.79082 \\
\hline 3.65 & 11.74338 & -178.78714 & -178.72536 & -178.44182 & -19.64605 & -20.07637 & -20.79084 \\
\hline 3.70 & 11.58469 & -178.78615 & -178.72535 & -178.44180 & -19.64528 & -20.07639 & -20.79086 \\
\hline 3.75 & 11.43023 & -178.78525 & -178.72534 & -178.44179 & -19.64452 & -20.07640 & -20.79087 \\
\hline 3.80 & 11.27983 & -178.78442 & -178.72534 & -178.44178 & -19.64376 & -20.07642 & -20.79088 \\
\hline 3.85 & 11.13334 & -178.78367 & -178.72533 & -178.44177 & -19.64302 & -20.07642 & -20.79089 \\
\hline 3.90 & 10.99060 & -178.78299 & -178.72533 & -178.44177 & -19.64228 & -20.07643 & -20.79090 \\
\hline 3.95 & 10.85148 & -178.78237 & -178.72533 & -178.44177 & -19.64155 & -20.07643 & -20.79090 \\
\hline 4.00 & 10.71584 & -178.78182 & -178.72533 & -178.44177 & -19.64082 & -20.07644 & -20.79090 \\
\hline 4.05 & 10.58354 & -178.78132 & -178.72534 & -178.44177 & -19.64011 & -20.07644 & -20.79091 \\
\hline 4.10 & 10.45448 & -178.78088 & -178.72534 & -178.44177 & -19.63939 & -20.07644 & -20.79091 \\
\hline 4.15 & 10.32852 & -178.78048 & -178.72534 & -178.44177 & -19.63869 & -20.07644 & -20.79091 \\
\hline 4.20 & 10.20556 & -178.78013 & -178.72535 & -178.44178 & -19.63799 & -20.07644 & -20.79091 \\
\hline 4.25 & 10.08550 & -178.77982 & -178.72535 & -178.44178 & -19.63730 & -20.07644 & -20.79090 \\
\hline 4.30 & 9.96822 & -178.77954 & -178.72536 & -178.44179 & -19.63662 & -20.07644 & -20.79090 \\
\hline 4.35 & 9.85364 & -178.77930 & -178.72536 & -178.44179 & -19.63594 & -20.07644 & -20.79090 \\
\hline 4.40 & 9.74167 & -178.77909 & -178.72537 & -178.44180 & -19.63527 & -20.07644 & -20.79090 \\
\hline 4.45 & 9.63221 & -178.77891 & -178.72537 & -178.44180 & -19.63462 & -20.07644 & -20.79090 \\
\hline 4.50 & 9.52519 & -178.77874 & -178.72538 & -178.44180 & -19.63397 & -20.07644 & -20.79090 \\
\hline 4.55 & 9.42052 & -178.77860 & -178.72538 & -178.44181 & -19.63333 & -20.07644 & -20.79090 \\
\hline 4.60 & 9.31812 & -178.77848 & -178.72538 & -178.44181 & -19.63270 & -20.07643 & -20.79089 \\
\hline 4.65 & 9.21793 & -178.77838 & -178.72539 & -178.44182 & -19.63207 & -20.07643 & -20.79089 \\
\hline 4.70 & 9.11986 & -178.77829 & -178.72539 & -178.44182 & -19.63146 & -20.07643 & -20.79089 \\
\hline 4.75 & 9.02386 & -178.77821 & -178.72540 & -178.44182 & -19.63086 & -20.07643 & -20.79089 \\
\hline 4.80 & 8.92987 & -178.77814 & -178.72540 & -178.44183 & -19.63027 & -20.07643 & -20.79089 \\
\hline
\end{tabular}


S-18

\begin{tabular}{llllllll}
\hline 4.85 & 8.83780 & -178.77809 & -178.72540 & -178.44183 & -19.62969 & -20.07643 & -20.79089 \\
4.90 & 8.74762 & -178.77804 & -178.72540 & -178.44183 & -19.62912 & -20.07643 & -20.79088 \\
4.95 & 8.65926 & -178.77800 & -178.72541 & -178.44184 & -19.62856 & -20.07643 & -20.79088 \\
5.00 & 8.57267 & -178.77796 & -178.72541 & -178.44184 & -19.62801 & -20.07643 & -20.79088 \\
\hline
\end{tabular}


Table S6 Absolute energies in hartrees of the $\mathrm{CN}$ decomposition for $\mathrm{N}_{2}$.

\begin{tabular}{|c|c|c|c|c|c|c|c|}
\hline$r(\AA)$ & $E^{\text {nuc }}$ & $\begin{array}{c}E^{\mathrm{cl}} \\
\mathrm{RHF}\end{array}$ & $\begin{array}{c}E^{\mathrm{cl}} \\
\mathrm{CASSCF}\end{array}$ & $\begin{array}{c}E^{\mathrm{cl}} \\
\mathrm{MRCI}\end{array}$ & $\begin{array}{l}E^{\text {noncl }} \\
\text { RHF }\end{array}$ & $\begin{array}{c}E^{\text {noncl }} \\
\text { CASSCF }\end{array}$ & $\begin{array}{c}E^{\text {noncl }} \\
\text { MRCI }\end{array}$ \\
\hline 0.50 & 51.85937 & -88.77445 & -88.73145 & -88.55824 & -14.68219 & -14.77355 & -15.23127 \\
\hline 0.55 & 47.14488 & -90.57392 & -90.52779 & -90.35411 & -14.51934 & -14.61760 & -15.07212 \\
\hline 0.60 & 43.21614 & -91.94103 & -91.89110 & -91.71637 & -14.34451 & -14.45088 & -14.90374 \\
\hline 0.65 & 39.89182 & -92.97376 & -92.91956 & -92.74371 & -14.17213 & -14.28773 & -14.73983 \\
\hline 0.70 & 37.04240 & -93.75224 & -93.69337 & -93.51651 & -14.00879 & -14.13470 & -14.58641 \\
\hline 0.75 & 34.57291 & -94.33881 & -94.27491 & -94.09728 & -13.85719 & -13.99444 & -14.44575 \\
\hline 0.80 & 32.41210 & -94.78087 & -94.71158 & -94.53347 & -13.71798 & -13.86761 & -14.31827 \\
\hline 0.85 & 30.50551 & -95.11403 & -95.03903 & -94.86069 & -13.59082 & -13.75381 & -14.20359 \\
\hline 0.90 & 28.81076 & -95.36499 & -95.28396 & -95.09710 & -13.47489 & -13.65223 & -14.10979 \\
\hline 0.95 & 27.29440 & -95.55372 & -95.46639 & -95.28056 & -13.36921 & -13.56186 & -14.01726 \\
\hline 1.00 & 25.92968 & -95.69524 & -95.60133 & -95.41647 & -13.27277 & -13.48169 & -13.93507 \\
\hline 1.05 & 24.69494 & -95.80087 & -95.70011 & -95.51609 & -13.18461 & -13.41073 & -13.86235 \\
\hline 1.10 & 23.57244 & -95.87915 & -95.77129 & -95.58797 & -13.10386 & -13.34811 & -13.79827 \\
\hline 1.15 & 22.54755 & -95.93659 & -95.82135 & -95.63859 & -13.02972 & -13.29306 & -13.74208 \\
\hline 1.20 & 21.60807 & -95.97814 & -95.85521 & -95.67288 & -12.96151 & -13.24492 & -13.69312 \\
\hline 1.25 & 20.74375 & -96.00760 & -95.87664 & -95.69464 & -12.89863 & -13.20312 & -13.65076 \\
\hline 1.30 & 19.94591 & -96.02788 & -95.88847 & -95.70677 & -12.84056 & -13.16721 & -13.61448 \\
\hline 1.35 & 19.20717 & -96.04121 & -95.89283 & -95.71147 & -12.78686 & -13.13683 & -13.58379 \\
\hline 1.40 & 18.52120 & -96.04929 & -95.89128 & -95.71040 & -12.73712 & -13.11172 & -13.55829 \\
\hline 1.45 & 17.88254 & -96.05341 & -95.88494 & -95.70481 & -12.69100 & -13.09170 & -13.53765 \\
\hline 1.50 & 17.28646 & -96.05454 & -95.87458 & -95.69558 & -12.64821 & -13.07670 & -13.52160 \\
\hline 1.55 & 16.72883 & -96.05340 & -95.86074 & -95.68338 & -12.60849 & -13.06668 & -13.50994 \\
\hline 1.60 & 16.20605 & -96.05055 & -95.84380 & -95.66869 & -12.57159 & -13.06160 & -13.50247 \\
\hline 1.65 & 15.71496 & -96.04640 & -95.82416 & -95.65192 & -12.53732 & -13.06134 & -13.49897 \\
\hline 1.70 & 15.25275 & -96.04128 & -95.80232 & -95.63347 & -12.50550 & -13.06555 & -13.49918 \\
\hline 1.75 & 14.81696 & -96.03541 & -95.77898 & -95.61381 & -12.47594 & -13.07361 & -13.50267 \\
\hline 1.80 & 14.40538 & -96.02900 & -95.75507 & -95.59347 & -12.44850 & -13.08459 & -13.50893 \\
\hline 1.85 & 14.01605 & -96.02219 & -95.73161 & -95.57309 & -12.42302 & -13.09737 & -13.51723 \\
\hline 1.90 & 13.64720 & -96.01511 & -95.70958 & -95.55335 & -12.39937 & -13.11080 & -13.52680 \\
\hline 1.95 & 13.29727 & -96.00785 & -95.68968 & -95.53483 & -12.37742 & -13.12393 & -13.53687 \\
\hline 2.00 & 12.96484 & -96.00049 & -95.67235 & -95.51799 & -12.35703 & -13.13605 & -13.54681 \\
\hline 2.05 & 12.64863 & -95.99309 & -95.65769 & -95.50309 & -12.33810 & -13.14679 & -13.55613 \\
\hline 2.10 & 12.34747 & -95.98572 & -95.64558 & -95.49020 & -12.32052 & -13.15599 & -13.56455 \\
\hline 2.15 & 12.06032 & -95.97840 & -95.63577 & -95.47926 & -12.30418 & -13.16370 & -13.57194 \\
\hline 2.20 & 11.78622 & -95.97118 & -95.62792 & -95.47011 & -12.28900 & -13.17003 & -13.57827 \\
\hline 2.25 & 11.52430 & -95.96407 & -95.62172 & -95.46256 & -12.27487 & -13.17516 & -13.58360 \\
\hline 2.30 & 11.27378 & -95.95711 & -95.61686 & -95.45636 & -12.26173 & -13.17928 & -13.58803 \\
\hline 2.35 & 11.03391 & -95.95031 & -95.61306 & -95.45131 & -12.24948 & -13.18257 & -13.59168 \\
\hline 2.40 & 10.80403 & -95.94368 & -95.61010 & -95.44722 & -12.23807 & -13.18520 & -13.59466 \\
\hline 2.45 & 10.58354 & -95.93723 & -95.60779 & -95.44390 & -12.22743 & -13.18728 & -13.59709 \\
\hline 2.50 & 10.37187 & -95.93097 & -95.60599 & -95.44120 & -12.21750 & -13.18894 & -13.59907 \\
\hline 2.55 & 10.16850 & -95.92491 & -95.60458 & -95.43902 & -12.20822 & -13.19027 & -13.60068 \\
\hline
\end{tabular}




\begin{tabular}{|c|c|c|c|c|c|c|c|}
\hline 2.60 & 9.97296 & -95.91904 & -95.60347 & -95.43724 & -12.19954 & -13.19133 & -13.60198 \\
\hline 2.65 & 9.78479 & -95.91338 & -95.60259 & -95.43579 & -12.19141 & -13.19218 & -13.60303 \\
\hline 2.70 & 9.60359 & -95.90791 & -95.60190 & -95.43460 & -12.18378 & -13.19286 & -13.60389 \\
\hline 2.75 & 9.42898 & -95.90264 & -95.60134 & -95.43362 & -12.17663 & -13.19342 & -13.60459 \\
\hline 2.80 & 9.26060 & -95.89757 & -95.60089 & -95.43282 & -12.16991 & -13.19387 & -13.60516 \\
\hline 2.85 & 9.09813 & -95.89269 & -95.60053 & -95.43215 & -12.16358 & -13.19424 & -13.60563 \\
\hline 2.90 & 8.94127 & -95.88800 & -95.60024 & -95.43159 & -12.15762 & -13.19454 & -13.60601 \\
\hline 2.95 & 8.78972 & -95.88349 & -95.59999 & -95.43113 & -12.15201 & -13.19479 & -13.60632 \\
\hline 3.00 & 8.64323 & -95.87916 & -95.59979 & -95.43074 & -12.14671 & -13.19499 & -13.60658 \\
\hline 3.05 & 8.50154 & -95.87500 & -95.59963 & -95.43041 & -12.14170 & -13.19516 & -13.60679 \\
\hline 3.10 & 8.36441 & -95.87100 & -95.59949 & -95.43013 & -12.13697 & -13.19530 & -13.60696 \\
\hline 3.15 & 8.23165 & -95.86716 & -95.59937 & -95.42989 & -12.13250 & -13.19542 & -13.60711 \\
\hline 3.20 & 8.10303 & -95.86346 & -95.59927 & -95.42969 & -12.12827 & -13.19551 & -13.60723 \\
\hline 3.25 & 7.97836 & -95.85990 & -95.59919 & -95.42952 & -12.12426 & -13.19560 & -13.60733 \\
\hline 3.30 & 7.85748 & -95.85647 & -95.59911 & -95.42937 & -12.12046 & -13.19568 & -13.60741 \\
\hline 3.35 & 7.74020 & -95.85317 & -95.59905 & -95.42923 & -12.11687 & -13.19573 & -13.60749 \\
\hline 3.40 & 7.62638 & -95.84999 & -95.59899 & -95.42912 & -12.11346 & -13.19579 & -13.60755 \\
\hline 3.45 & 7.51585 & -95.84693 & -95.59894 & -95.42901 & -12.11022 & -13.19585 & -13.60761 \\
\hline 3.50 & 7.40848 & -95.84397 & -95.59889 & -95.42892 & -12.10715 & -13.19589 & -13.60767 \\
\hline 3.55 & 7.30414 & -95.84112 & -95.59885 & -95.42883 & -12.10423 & -13.19594 & -13.60771 \\
\hline 3.60 & 7.20269 & -95.83838 & -95.59880 & -95.42875 & -12.10146 & -13.19598 & -13.60776 \\
\hline 3.65 & 7.10402 & -95.83573 & -95.59876 & -95.42868 & -12.09881 & -13.19602 & -13.60781 \\
\hline 3.70 & 7.00802 & -95.83319 & -95.59872 & -95.42861 & -12.09629 & -13.19606 & -13.60785 \\
\hline 3.75 & 6.91458 & -95.83074 & -95.59868 & -95.42854 & -12.09388 & -13.19609 & -13.60789 \\
\hline 3.80 & 6.82360 & -95.82840 & -95.59864 & -95.42848 & -12.09157 & -13.19613 & -13.60793 \\
\hline 3.85 & 6.73498 & -95.82615 & -95.59861 & -95.42842 & -12.08936 & -13.19617 & -13.60797 \\
\hline 3.90 & 6.64864 & -95.82399 & -95.59857 & -95.42837 & -12.08723 & -13.19620 & -13.60801 \\
\hline 3.95 & 6.56448 & -95.82194 & -95.59853 & -95.42831 & -12.08518 & -13.19624 & -13.60804 \\
\hline 4.00 & 6.48242 & -95.81997 & -95.59850 & -95.42826 & -12.08320 & -13.19627 & -13.60808 \\
\hline 4.05 & 6.40239 & -95.81811 & -95.59846 & -95.42821 & -12.08128 & -13.19630 & -13.60811 \\
\hline 4.10 & 6.32431 & -95.81634 & -95.59843 & -95.42817 & -12.07942 & -13.19633 & -13.60815 \\
\hline 4.15 & 6.24812 & -95.81466 & -95.59839 & -95.42812 & -12.07760 & -13.19636 & -13.60818 \\
\hline 4.20 & 6.17373 & -95.81307 & -95.59836 & -95.42808 & -12.07583 & -13.19639 & -13.60821 \\
\hline 4.25 & 6.10110 & -95.81157 & -95.59833 & -95.42805 & -12.07410 & -13.19642 & -13.60823 \\
\hline 4.30 & 6.03016 & -95.81017 & -95.59831 & -95.42801 & -12.07241 & -13.19644 & -13.60826 \\
\hline 4.35 & 5.96085 & -95.80884 & -95.59828 & -95.42798 & -12.07074 & -13.19646 & -13.60828 \\
\hline 4.40 & 5.89311 & -95.80761 & -95.59826 & -95.42795 & -12.06911 & -13.19648 & -13.60830 \\
\hline 4.45 & 5.82690 & -95.80645 & -95.59824 & -95.42792 & -12.06750 & -13.19650 & -13.60832 \\
\hline 4.50 & 5.76215 & -95.80537 & -95.59822 & -95.42790 & -12.06592 & -13.19652 & -13.60834 \\
\hline 4.55 & 5.69883 & -95.80436 & -95.59820 & -95.42788 & -12.06435 & -13.19653 & -13.60835 \\
\hline 4.60 & 5.63689 & -95.80343 & -95.59819 & -95.42786 & -12.06281 & -13.19655 & -13.60837 \\
\hline 4.65 & 5.57628 & -95.80257 & -95.59817 & -95.42784 & -12.06128 & -13.19656 & -13.60838 \\
\hline 4.70 & 5.51695 & -95.80177 & -95.59816 & -95.42783 & -12.05977 & -13.19657 & -13.60839 \\
\hline 4.75 & 5.45888 & -95.80103 & -95.59815 & -95.42782 & -12.05828 & -13.19658 & -13.60840 \\
\hline 4.80 & 5.40202 & -95.80035 & -95.59814 & -95.42780 & -12.05680 & -13.19659 & -13.60841 \\
\hline
\end{tabular}




\begin{tabular}{llllllll}
\hline 4.85 & 5.34633 & -95.79973 & -95.59813 & -95.42779 & -12.05534 & -13.19659 & -13.60841 \\
4.90 & 5.29177 & -95.79916 & -95.59812 & -95.42779 & -12.05389 & -13.19660 & -13.60842 \\
4.95 & 5.23832 & -95.79864 & -95.59812 & -95.42778 & -12.05246 & -13.19660 & -13.60842 \\
5.00 & 5.18594 & -95.79816 & -95.59811 & -95.42777 & -12.05104 & -13.19661 & -13.60843 \\
\hline
\end{tabular}




\section{Sample input file}

The following input file performs RHF, CASSCF, and MRCI calculations for $\mathrm{H}_{2}$ at a bond distance of $1.00 \AA$.

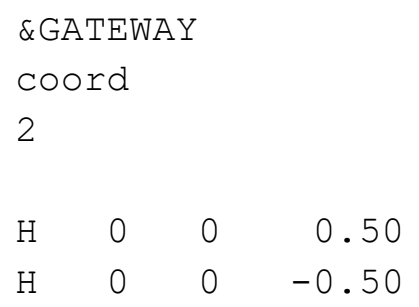


Spin=1

CIAl $I=1$

$\& M R C I$ 


\section{Key modifications to the OpenMolcas source code and the Python script for energy calculation}

Modifications were performed on the following two OpenMolcas source files:

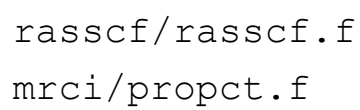

We exported some working variables using the HDF5 interface provided by OpenMolcas. Some of the key subroutines we used are mh5_create_file, mh5_create_dset_real, mh5_put_dset_array_real,mh5_close_dset, and mh5_close_file. The subroutines used for exporting working variables were inserted at the end of the calculation and before the memory is freed. Below is a list of important variables that we utilized for this study.

\begin{tabular}{|c|c|c|c|}
\hline File & Variable & Dimension & Meaning \\
\hline rasscf/rasscf.f & Work (lD1A) & NTOT2 & $\begin{array}{l}\text { 1-RDM of CASSCF/RASSCF } \\
\text { in the atomic orbital basis for } \\
\text { the active electrons }\end{array}$ \\
\hline rasscf/rasscf.f & Work ( ID1 I ) & NTOT2 & $\begin{array}{l}\text { 1-RDM of CASSCF/RASSCF } \\
\text { in the atomic orbital basis for } \\
\text { the inactive electrons }\end{array}$ \\
\hline rasscf/rasscf.f & PotNuc & 1 & Nuclear repulsion energy \\
\hline mrci/propct.f & Work ( LDAO) & $\mathrm{NBAST} * \star 2$ & $\begin{array}{l}1-\mathrm{RDM} \text { of } \mathrm{MRCI} \text { in the atomic } \\
\text { orbital basis }\end{array}$ \\
\hline
\end{tabular}

The CASSCF 1-RDM can be obtained by adding Work (ID1A) and Work (IDII) elementwise. The one-electron integral was retrieved by calling the subroutine RdOne, and the two-electron integral was retrieved by calling subroutine RdOrd. The integrals were also exported using the HDF5 interface.

After exporting the respective matrices, the resulting HDF5 data files were then read by a Python script using the h5py module and converted to the proper dimensions. Matrices stored in triangular form were converted back to square matrices prior to calculating the energy.

Subsequent calculations were performed using the numpy module.

The following code calculates the energy components of the three decomposition schemes. The variable $\mathrm{H}$ contains the one-electron integrals, D is the 1-RDM, TwoInt contains the twoelectron integrals, Etot is the total electronic energy, and Enuc is the nuclear repulsion energy.

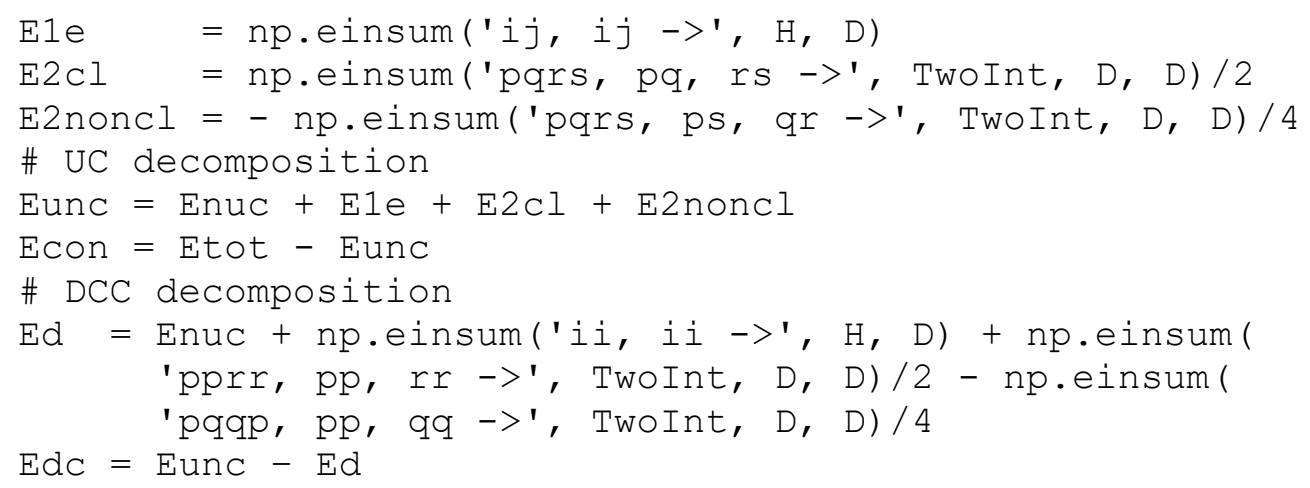


\# CN decomposition

$\mathrm{Ecl}=\mathrm{Enuc}+\mathrm{E} 1 \mathrm{e}+\mathrm{E} 2 \mathrm{Cl}$

Enoncl $=$ E2noncl + Econ 